\title{
Titan's Upper Atmosphere: Structure and Ultraviolet Emissions
}

\author{
Darrell F. Strobel \\ Department of Earth and Planetary Sciences, and Department of Physics and Astronomy. \\ The Johns Hopkins University, Baltimore, Maryland 21218 \\ Michael E. Summers \\ E. O. Hulbart Center for Space Research, Naval Research Laboratory, Washington, DC 20375 \\ AND \\ XUN ZHU \\ Department of Earth and Planetary Sciences, The Johns Hopkins University, Baltimore, Maryland 21218 \\ Received April 21, 1992; revised August 18. 1992
}

The Voyager 1 ultraviolet spectrometer (UVS) solar occultation and airglow data obtained during the Titan flyby are analyzed for composition and thermal structure of the upper atmosphere and relative importance of airglow excitation processes. From optical depth profiles inferred by Smith et al. (1982, J. Geophys. Res. 87, 1351-1359) for the evening terminator, the entrance occultation, an asymptotic temperature of $T_{\infty}=175 \mathrm{~K}$, and a $\mathrm{CH}_{4}$ mixing ratio increasing from $0.06 \pm 0.01$ at $1000 \mathrm{~km}$ to $0.20 \pm 0.02$ at $1400 \mathrm{~km}$ are derived. The optical depth profiles are not of sufficient accuracy to extrapolate and accurately infer the tropopause $\mathrm{CH}_{4}$ mixing ratio, but our preference is for high values $\sim 0.026-0.034$. The homopause occurs high in the atmosphere $(\sim 1000 \mathrm{~km})$ with an eddy diffusion coefficient $K_{0} \sim(4-8) \times 10^{8} \mathrm{~cm}^{2} \mathrm{sec}^{-1}$ at this level and an approximate altitude dependence $K=K_{0} \exp (\zeta / 1.6)$, where $\zeta$ is the normalized geopotential height.

From the shape of the observed bright limb profile for $\mathrm{N}_{2}$ emission (924-998 $\AA$ ) the magnetospheric power dissipation is, at most, $10 \%$ of the solar EUV power input to Titan's extended atmosphere ( $=2 \times 10^{9} \mathrm{~W}$ for $\lambda<800 \AA$ ) and consistent with our estimates of magnetospheric power delivered to the ionopause by curvature drift of thermal and suprathermal electrons. This value of magnetospheric power input is a factor of 25 lower than the original estimate of Strobel and Shemansky (1982, J. Geophys. Res. 87, 1361-1368). Our calculated absolute intensities for the $\mathrm{N}^{+}(1085 \AA)$ multiplet are consistent with the UVS observed intensities after downward revision by the Holberg et al. (1982, Astrophys. $J$., 257, 656-671) inflight calibration scheme, whereas the calculated $N_{2} c_{4}^{\prime}(0-0,958$ $\AA$ ) plus (0-1, 981 $\AA$ ) band intensities (the 970- $\AA$ feature) are only $\sim 50 \%$ of the observed values. The nonthermal $N$ atom escape rate is estimated to be $\leq 10^{25} \mathrm{sec}^{-1}$.

An upper limit on the tropopause Ar mixing ratio of 0.14 is derived from a comparison of the calculated relative intensities of the Ar resonance lines at 1048 and $1067 \AA$ with the $\mathrm{N}^{+}(1085-\AA)$ multiplet to the observationally inferred upper limit of 0.5 . 1992 Academic Press, Inc.

\section{INTRODUCTION}

The structure of Titan's thermosphere depends almost exclusively on the interpretation of data obtained by the Ultraviolet Spectrometer Experiment (UVS) on the Voyager 1 spacecraft during its Titan flyby in November 1980 (Broadfoot et al. 1981, Smith et al. 1982). In contrast, the thermal structure of the troposphere and stratosphere is derived from Voyager IRIS and radio occultation data (Lellouch et al. 1989). The connection between the upper stratosphere and the thermosphere is weakly constrained by groundbased observations of Titan's occultation of 28 Sagittarii (Hubbard et al. 1990, Sicardy et al. 1990) and must rely on the detailed, physically based radiative transfer model of Yelle (1991).

Broadfoot et al. (1981) and Strobel and Shemansky (1982) interpreted EUV emissions observed by the UVS as electron impact excitation of $\mathrm{N}_{2}$ in the upper thermosphere due primarily to precipitation of magnetospheric electrons in Titan's sunlit hemisphere and estimated a power dissipation of $\sim 5 \times 10^{9} \mathrm{~W}$. In a recent paper Strobel et al. (1991) calculated the disk center and bright limb intensities of $\mathrm{N}_{2}$ and $\mathrm{N}^{+}$UV emission features due to solar and photoelectron excitation of $\mathrm{N}_{2}$. When they adopted the inflight calibration scheme developed by Holberg et al. $(1982,1991)$ which yielded a downward revision of the 
originally reported intensities below $1100 \AA$ by a factor of $\sim 1.6$, they found that the $\mathrm{N}^{+}(1085-\AA)$ multiplet and the $\mathrm{N}_{2}$ LBH intensities could be explained by solar and photoelectron excitation of $\mathrm{N}_{2}$. This would imply that magnetospheric electrons play only a minor role in Titan's UV airglow. However, if the bright $970-\AA$ feature is mostly $\mathrm{N}_{2} c_{4}^{\prime}$-band emission, then the calculated photoelectron excitation rate is only $40 \%$ of the observed intensity and would suggest that magnetospheric electrons contribute significantly to the observed EUV emission.

In this paper we extend the study of Strobel et al. (1991) to explicitly estimate the power dissipation by magnetospheric electrons in Titan's upper atmosphere in a manner similar to the analysis of Strobel et al.(1990) for Triton's upper atmosphere. With this estimated power dissipation rate, magnetospheric electron excitation rates of $\mathrm{N}_{2}$ are calculated for Titan by the same method used by Strobel et al. (1991) for Triton. The resulting disk center and bright limb intensities for the most important nitrogen airglow features are computed based on energetic magnetospheric electron fluxes observed during the Titan flyby (Hartle $e t$ al. 1982), Maclennan et al. 1982).

We discovered that the model thermosphere given in Smith et al. (1982) was not consistent with the observed optical depth profiles given in their Fig. 3. Thus the first task was to derive a new model thermosphere which smoothly joins the Yelle (1991) Model-A pressure and the temperature profiles at lower altitudes and has tangential optical depths in agreement with those inferred from the UVS solar occultation data by Smith et al. (1982).

\section{MODEL ATMOSPHERE}

Optical depths at six wavelengths, as a function of radial distance from the center of Titan $\left(r_{\mathrm{T}}=2575 \mathrm{~km}\right.$ is the surface) for the entrance occultation at the evening terminator, were digitized from Fig. 3 of Smith et al. (1982). Each profile represents the effective optical depth averaged over $9.26 \AA$ whose central value is the indicated wavelength. The optical depth at $725 \AA$ was assumed to be due solely to $\mathrm{N}_{2}$ absorption by Smith et al. (1982), although as we shall show $\mathrm{CH}_{4}$ contributes significantly. Methane absorption contributes also to the optical depths at $928,1114,1151$, and $1373 \AA$, whereas the optical depth at $1540 \AA$ must be due to other hydrocarbons and organic molecules, such as $\mathrm{C}_{2} \mathrm{H}_{x}$ hydrocarbons and simple nitriles. In addition these molecules will contribute to the observed optical depths at shorter wavelengths. However, based on the photochemical model of Yung et al. (1984) and hydrocarbon absorption cross sections given in Table I, most of the opacity in the $928,1114,1151$, and 1373- $\AA$ channels should be due to $\mathrm{CH}_{4}$. The cross sections in Table I are derived from the database used by the Voyager UVS team to analyze occultation data. It should be noted that $\mathrm{N}_{2}$ exhibits band structure in absorption at $725 \AA$ and from low spectral resolution data one infers an average cross section over the $9.26-\AA$ channel of $3.5 \times 10^{-17} \mathrm{~cm}^{2}$. If the individual rotational lines in these bands are extremely narrow, this average cross section is too large and the underlying continuum cross section will be more appropriate. Smith et al.(1982) used the continuum value to infer the reference $\mathrm{N}_{2}$ density at $z=1265 \mathrm{~km}(r=3840$ $\mathrm{km}$ ), and this value was used in our analysis.

Based on the authors' experience with UVS solar occultation data from other encounters, optical depths, $\tau$, are most accurately inferred in the interval of $0.15-1.9$. Thus the four channels, $725,928,1114$, and 1151 , yield the most reliable information on composition, density, and temperature in the range $z=925-1325 \mathrm{~km}(r=3500-3900$ $\mathrm{km})$.

A background model atmosphere for $\mathrm{N}_{2}$ density and temperature profiles was constructed based on the Lellouch et al.(1989) model atmosphere for the troposphere and stratosphere with the Yelle (1991) Model A pressure and temperature profiles imposed at $z=293 \mathrm{~km}$ to represent the mesosphere and lower thermosphere. The upper thermosphere is described by the Bates' (1959) temperature expression with the asymptotic temperature $\left(T_{\infty}\right)$ constrained by the UVS optical depth profiles. A model atmosphere with $T_{\infty}=185.4 \mathrm{~K}$ yields tangential $\mathrm{N}_{2}$ column densities which with the $\mathrm{N}_{2}$ continuum absorption cross section of $2.5 \times 10^{-17} \mathrm{~cm}^{2}$ accurately fits the inferred optical depth profile at $725 \AA$ if the atmosphere were devoid of hydrocarbons.

To model the $\mathrm{CH}_{4}$ mixing ratio the analytic expression of Steiner and Bauer (1990) was adopted,

$$
f_{\mathrm{CH}_{4}}=\frac{A}{\left[\mathrm{~N}_{2}\right]_{0}}\{1+\exp (\zeta(1-\kappa))\}^{(3 /(7(1-\kappa)))}+\frac{7 \phi N}{3 K_{0}\left[\mathrm{~N}_{2}\right]_{0}},
$$

where $\zeta=1.76 \times 10^{5} \mathrm{~km} \cdot\left(z-z_{0}\right) /\left(r_{0}\left(r_{\mathrm{T}}+z\right)\right)$ is the normalized geopotential height measured relative to the homopause $\left(r_{0}=r_{\mathrm{T}}+z_{0}\right), \boldsymbol{A}$ is an integration constant, $\left[\mathrm{N}_{2}\right]_{0}$ is the $\mathrm{N}_{2}$ density at the homopause, $\phi$ is the upward flux of $\mathrm{CH}_{4}$ referenced to Titan's surface, $H$ is the scale height of the atmosphere, and $K=K_{0} \exp (\kappa \zeta)$ is the vertical eddy diffusion coefficient with magnitude $K_{0}$ at the homopause.

Vertical mixing rates in Titan's upper atmosphere are inferred from boundary conditions on the $\mathrm{CH}_{4}$ and the sum of the heavier hydrocarbons' mixing ratios at the tropopause and at $z=1125 \mathrm{~km}$ in the thermosphere. The basic approach and methodology were developed in Smith et al. (1982), who derived the homopause location at $z_{0}=$ $925 \pm 70 \mathrm{~km}$, with $K_{0}=1_{-0.7}^{+2} \times 10^{8} \mathrm{~cm}^{2} \mathrm{sec}^{-1}$. They suggested that the vertical eddy coefficient decreases with altitude as $\sim\left[\mathrm{N}_{2}\right]^{-0.67}$. In a more detailed analysis with photochemistry using the same boundary conditions Yung 
TABLE I

Absorption Cross Sections Used in Optical Depth Calculations

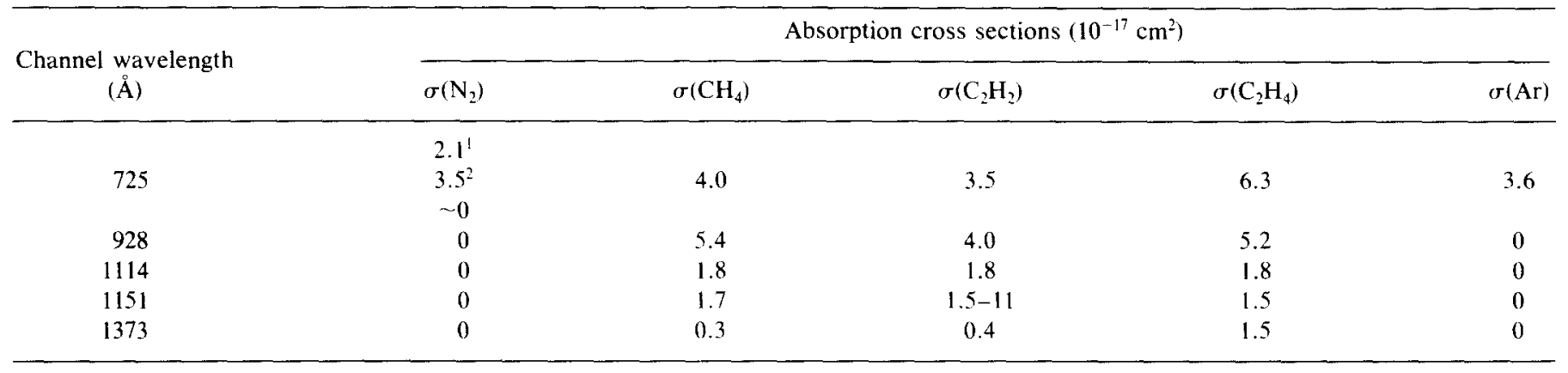

${ }^{1}$ Continuum cross section.

${ }^{2}$ Averaged cross section over 9.26- $\AA$ channel.

et al. (1984) found that the vertical eddy diffusion coefficient increased with height as $\left[\mathrm{N}_{2}\right]^{-0.5}$, up to a homopause located at $z_{0}=680 \mathrm{~km}$ with constant $K=1 \times 10^{6} \mathrm{~cm}^{2}$ $\sec ^{-1}$ above it. Steiner and Bauer (1990) repeated the analysis of Smith et al. (1982) and inferred an eddy diffusion coefficient proportional to $\left[\mathrm{N}_{2}\right]^{-0.51}$ and equal to $1 \times$ $10^{6} \mathrm{~cm}^{2} \mathrm{sec}^{-1}$ at an inferred homopause $z_{0}=660 \mathrm{~km}$.

With the homopause inferred by Smith et al. (1982) at $z_{0}=925 \mathrm{~km}\left(r_{0}=3500 \mathrm{~km}\right), K_{0}=2 \times 10^{8} \mathrm{~cm}^{2} \mathrm{sec}^{-1}$ in our adopted model atmosphere. With $\kappa=0$, a constant $K$ profile, expression (1) becomes, for the $\mathrm{CH}_{4}$ mixing ratio in the Smith et al. (1982) model,

$$
f_{\mathrm{CH}_{4}}=0.020\{1+\exp (\zeta)\}^{3 / 7}+0.006,
$$

and Fig. 1 illustrates how well the resulting model atmosphere fits the inferred optical depths. A close examination of Fig. 1 reveals that the only real agreement is in a small altitude region (near $z=1125 \mathrm{~km}, r=3700 \mathrm{~km}$ ) for the channels $928,1114,1151$, and $1373 \AA$ whose optical depths are dominated by $\mathrm{CH}_{4}$ opacity. Based on the absorption cross sections in Table I and the inferred optical depths, one can conclude that the channels dominated by $\mathrm{CH}_{4}$ are not internally consistent, in particular the channel 1114- $\AA$ optical depth appears to be $50 \%$ larger relative to what would be expected based on the other channels. Smith et al. (1982) preferred channel $928 \AA$ where the $\mathrm{CH}_{4}$ absorption cross section is three times larger than channels 1114 and $1151 \AA$ to infer its density profile. The predicted optical depth profile at $725 \AA$ exceeds significantly the observations due to the $\mathrm{CH}_{4}$ contribution. The contribution by only $\mathrm{N}_{2}$ would yield almost a perfect fit to the data, which was the assumption that Smith et al.(1982) used to infer the $\mathrm{N}_{2}$ density and temperature at $z=1165$ $\mathrm{km}(r=3840 \mathrm{~km})$.

The contributions of the higher hydrocarbons $\mathrm{C}_{2} \mathrm{H}_{x}$ were included with the Steiner and Bauer expression,

$$
f_{\mathrm{C}_{2} \mathrm{H}_{x}}=B\left\{\{1+\exp (\zeta(1-\kappa))\}^{-1 /(14(1-\kappa))}-1\right\},
$$

with $B=-0.1$ and $\kappa=0$. Whereas this expression yields heavier hydrocarbon mixing ratios greater than $1 \%$ above $3600 \mathrm{~km}$, it clearly underestimates their observed mixing ratio in the lower stratosphere by a factor of $10^{4}$.

In Fig. 2 the optical depth profiles corresponding to the Steiner and Bauer (1990) model atmosphere are compared with the inferred UVS optical depths. (Note that for eddy diffusion to equal molecular diffusion at the homopause, $z_{0}=660 \mathrm{~km}$, in our adopted model atmosphere $K_{0}=2 \times$ $10^{6} \mathrm{~cm}^{2} \mathrm{sec}^{-1}$, with $\kappa=0.51$.) The fit to channels 928 and $1151 \AA$ is significantly better than the Smith et al. (1982) model atmosphere in Fig. 1, but the calculated optical depth profile at $725 \AA$ is still larger than the data, indicative of too large $\mathrm{N}_{2}$ densities in the upper thermosphere. Also

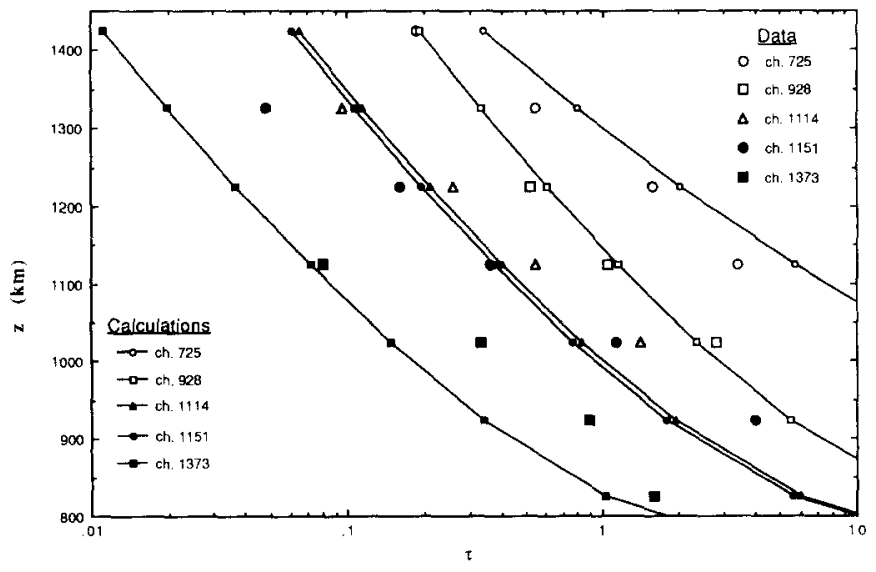

FIG. 1. Comparison of calculated and observationally inferred optical depth profiles for indicated wavelength channels in angstroms at evening terminator, entrance occultation. Large symbols denote data and small symbols connected by lines denote calculations with the Smith et al. (1982) model atmosphere. 


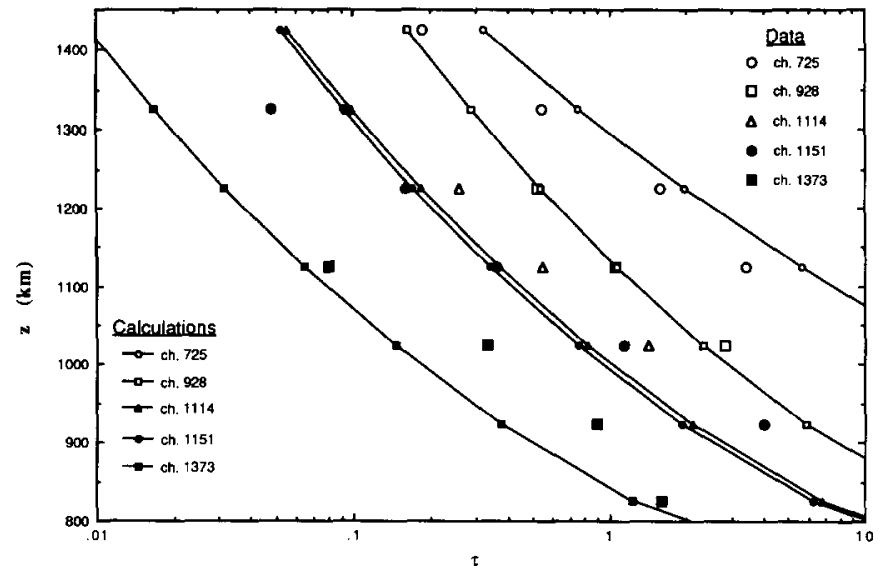

FIG. 2. Same as Fig. 1, but for the Steiner and Bauer (1990) model atmosphere.

the slope of the calculated optical depth profiles at 928 , 1114 , and $1151 \AA$ is shallower than the data, which is also true for the Smith et al. (1982) profiles. While both models satisfy the observational constraint at $z=1125 \mathrm{~km}(r=$ $3700 \mathrm{~km}$ ), neither captures the essentially constant mixing ratio implied by the data between $z=925-1125 \mathrm{~km}$ and the smaller $\mathrm{CH}_{4}$ scale height implied above $z=1125 \mathrm{~km}$ than appropriate for $\mathrm{CH}_{4}$ in diffusive equilibrium.

To obtain a substantially better fit to the inferred optical depths, there are three essential requirements: (1) $\mathrm{CH}_{4}$ must be mixed to higher altitudes which implies a larger eddy diffusion coefficient at the homopause which is located at a higher altitude in the atmosphere than previously thought, (2) the $\mathrm{CH}_{4}$ mixing ratio at the tropopause must be higher than 0.017 as adopted by Steiner and Bauer (1990) in order to obtain the quasi-mixed distribution below $z=1125 \mathrm{~km}$, at which level the $\mathrm{CH}_{4}$ mixing ratio is $\sim 8 \%$, and (3) the asymptotic isothermal temperature, $T_{\infty}$, must be lower than $185.4 \mathrm{~K}$ to lower the $\mathrm{N}_{2}$ density in the upper thermosphere.

Three additional model atmospheres were produced to illustrate these requirements. In addition, argon was introduced in the model atmospheres to satisfy the constraint on mean molecular mass $(27.8<\bar{m}<29.3 \mathrm{amu})$ inferred by Lellouch et al. (1989). The first model atmosphere has a temperature profile with $T_{\infty}=172 \mathrm{~K}$, homopause $z_{0}=$ $985 \mathrm{~km}$ with $K_{0}=4 \times 10^{8} \mathrm{~cm}^{2} \mathrm{sec}^{-1}, \kappa=0.625$, tropopause $\mathrm{CH}_{4}$ and Ar mixing ratios $=0.026$ and 0.014 , respectively, $\bar{m}=27.8 \mathrm{amu}, \mathrm{CH}_{4}$ and $\mathrm{Ar}$ mixing ratios given by

$$
\begin{aligned}
f_{\mathrm{CH}_{4}} & =0.02\{1+\exp (0.325 \zeta)\}^{3 / 7(1-\kappa)}+0.006 \\
f_{\mathrm{Ar}} & =0.014\{1+\exp (0.325 \zeta)\}^{-0.3 /(1-\kappa)},
\end{aligned}
$$

and $B=-0.1$ in expression (3) for $f_{\mathrm{C}_{2} \mathrm{H}_{\mathrm{x}}}$. As shown in Fig. 3 , this model atmosphere yields optical depth profiles with slopes that are a significant improvement over the previous two models in channels dominated by $\mathrm{CH}_{4}$ absorption, but is slightly too opaque at $725 \AA$. The improvement in the fit to channel $1373 \AA$ is primarily due to the adoption of the relative proportions of $\mathrm{C}_{2} \mathrm{H}_{2}$ and $\mathrm{C}_{2} \mathrm{H}_{4}$ from the Yung et al. (1984) photochemical model to obtain an average cross section of $1.1 \times 10^{-17} \mathrm{~cm}^{2}$ for this channel rather than the assumption of pure $\mathrm{C}_{2} \mathrm{H}_{2}$ for the $\mathrm{C}_{2} \mathrm{H}_{x}$ mixing ratio.

The second model atmosphere is constrained by the upper limit on the tropopause temperature, $74.5 \mathrm{~K}$, which limits the stratospheric $\mathrm{CH}_{4}$ mixing ratio to at most $\mathbf{0 . 0 3 4}$ (Samuelson et al. 1981, Lellouch et al. 1989). The inferred atmosphere has $T_{\infty}=175 \mathrm{~K}$. The temperature difference from the previous model with $T_{\infty}=172 \mathrm{~K}$ is not significant, but the slightly larger $T_{\infty}$ ensures that the derived thermosphere merges smoothly with the adopted lower model atmosphere at $z=900 \mathrm{~km}$. The homopause is now at $z_{0}=1035 \mathrm{~km}, K_{0}=8.4 \times 10^{8} \mathrm{~cm}^{2} \mathrm{sec}^{-1}, \kappa=0.625$, tropopause Ar mixing ratio $=0.136$, and $\bar{m}=28.7 \mathrm{amu}$. Expressions (4) for $\mathrm{CH}_{4}$ and $\mathrm{Ar}$ become

$$
\begin{aligned}
f_{\mathrm{CH}_{4}} & =0.024\{1+\exp (0.325 \zeta)\}^{0.69}+0.01 \\
f_{\mathrm{Ar}} & =0.136\{1+\exp (0.325 \zeta)\}^{-0.48} .
\end{aligned}
$$

The calculated optical depth profiles for this model atmosphere are shown in Fig. 4 and indicate an excellent fit to the $725-\AA$ channel. The data point at $z=1125 \mathrm{~km}$, where $\tau(725 \AA)=3.4$ corresponds to weak transmission of sunlight through Titan's atmosphere $\sim 0.03$, and actually may be scattered light in the spectrometer, which was a substantial problem as discussed by Smith et al. (1982), and accordingly not weighted heavily in this analysis. Likewise the data point for channel $1373 \AA$ at $825 \mathrm{~km}$

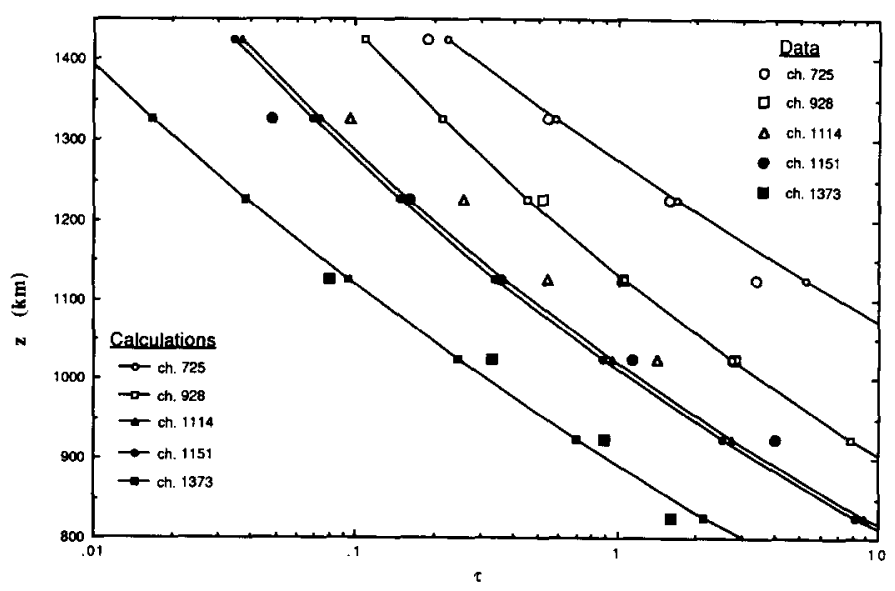

FIG. 3. Same as Fig. 1, but for the $2.6 \% \mathrm{CH}_{4}, 1.4 \%$ Ar model atmosphere. 


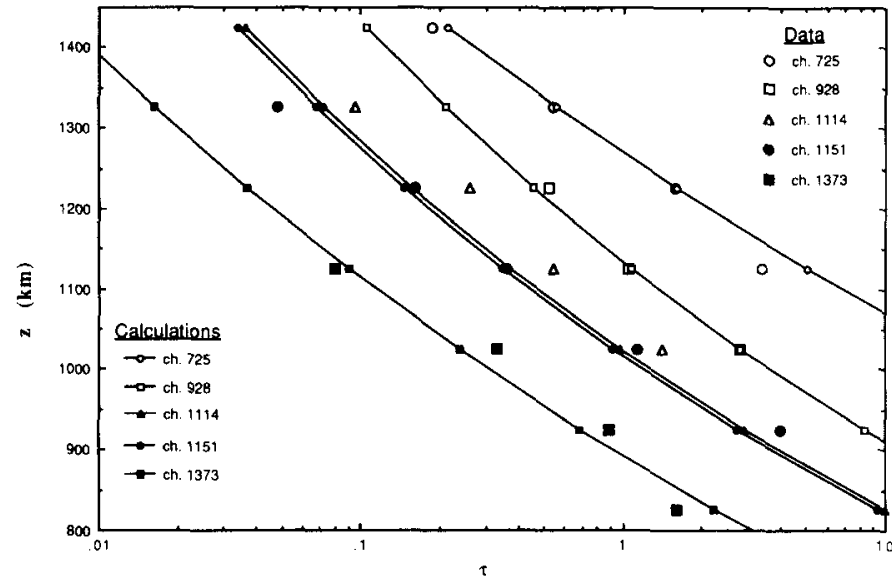

FIG. 4. Same as Fig. 1, but for the $3.4 \% \mathrm{CH}_{4}, 13.6 \%$ Ar model atmosphere.

suffers from the same problem. The $\mathrm{CH}_{4}$ channels are fit slightly better with this model than with the $2.6 \% \mathrm{CH}_{4}$ model in Fig. 3.

The last model considered also has $T_{\infty}=175 \mathrm{~K}$, the homopause at $z_{0}=1125 \mathrm{~km}, K_{0}=2.6 \times 10^{9} \mathrm{~cm}^{2} \mathrm{sec}^{-1}$, $\kappa=0.625$, tropopause $\mathrm{CH}_{4}$ and $\mathrm{Ar}$ mixing ratios of 0.05 and 0.1 , respectively, and $\bar{m}=28.2 \mathrm{amu}$. Expressions (4) for $\mathrm{CH}_{4}$ and $\mathrm{Ar}$ become

$$
\begin{aligned}
f_{\mathrm{CH}_{4}} & =0.03\{1+\exp (0.325 \zeta)\}^{0.69}+0.02 \\
f_{\mathrm{Ar}} & =0.1\{1+\exp (0.325 \zeta)\}^{-0.48} .
\end{aligned}
$$

This model, which violates the cold trap tropopause temperature, provides an equally excellent fit to the $725-\AA$ channel as shown in Fig. 5 and yields a somewhat better fit to channel $1151 \AA$ at the expense of a slightly worse fit

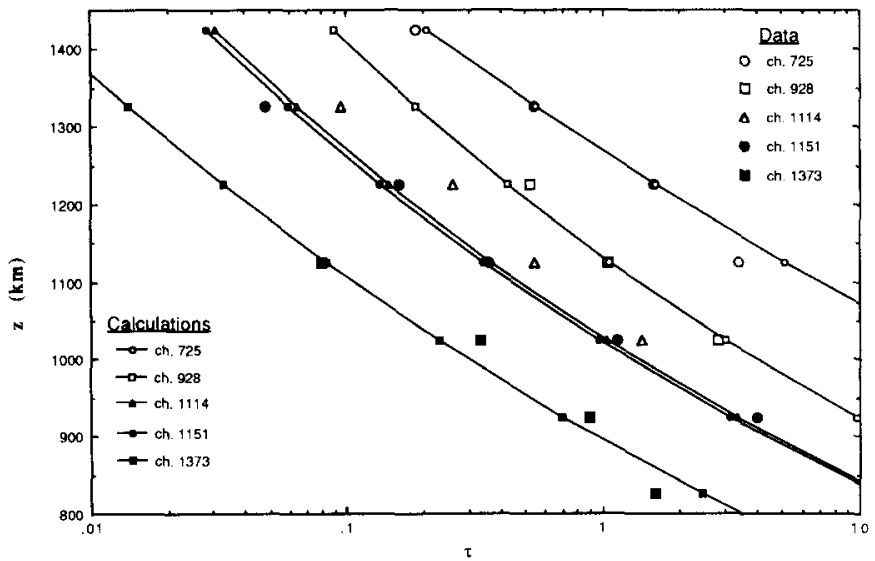

FIG. 5. Same as Fig. 1, but for the $5.0 \% \mathrm{CH}_{4}, 10 \%$ Ar model atmosphere.

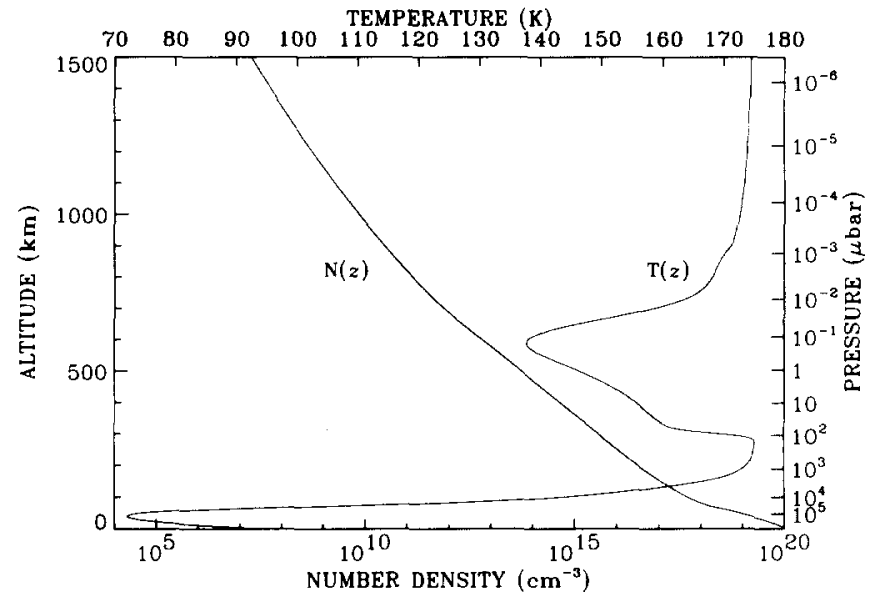

FIG. 6. Our preferred model atmosphere with $T_{\infty}=175 \mathrm{~K}$, number density, and temperature as a function of altitude and pressure.

to the $928-\AA$ channel when compared to the $3.4 \% \mathrm{CH}_{4}$ model in Fig. 4. In our opinion, all three models with $T_{\infty} \sim 172-175 \mathrm{~K}$ and $\mathrm{CH}_{4} \sim 0.026-0.05$ are as close to the optimal fit as is worth pursuing in this study, given the quality of the analyzed data. The $3.4 \%$ model is probably overall a slightly better fit to the data. In Fig. 6 our preferred $T_{\infty}=175 \mathrm{~K}$ model atmosphere, consistent with the inferred $T_{\infty}=176 \pm 20 \mathrm{~K}$ by Smith et al. (1982), is illustrated and was used for the models in Figs. 4-5. Note that the number density profile is the sum of the $\mathrm{N}_{2}, \mathrm{CH}_{4}$, and $\mathrm{Ar}$ concentrations. In Fig. 7 the various $\mathrm{Ar}$ and $\mathrm{CH}_{4}$ mixing ratio profiles are shown.

The value of $K$ at the homopause, $K_{0}=(0.4-2.6) \times 10^{9}$ $\mathrm{cm}^{2} \mathrm{sec}^{-1}$, is so extremely large that one might be tempted to regard it as ridiculous. If we attribute vertical mixing to saturation of internal gravity waves and adopt the pa-

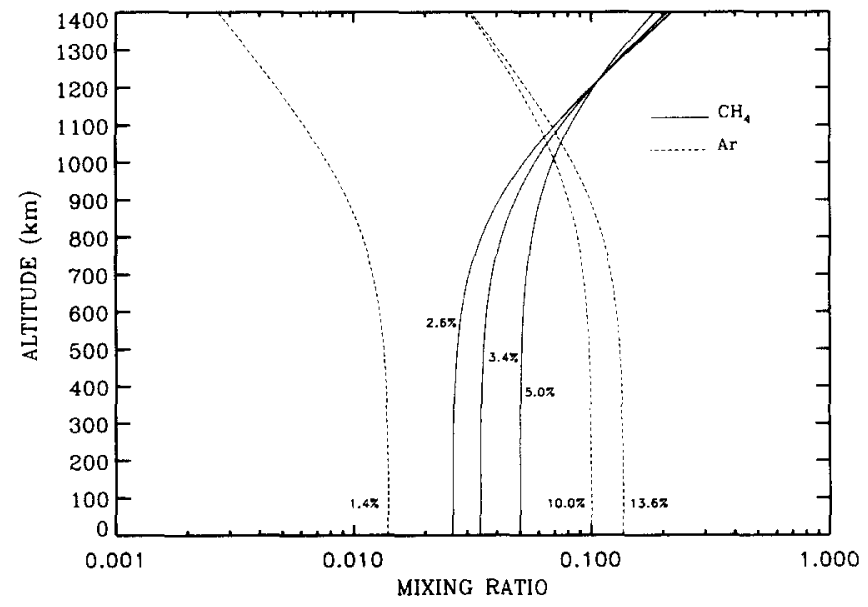

FIG. 7. The volume mixing ratios of $\mathrm{CH}_{4}$ and $\mathrm{Ar}$ as function of altitude for the indicated tropopause boundary values. 
rameterization developed by Lindzen (1981), who derived the gravity wave induced eddy diffusion coefficient for momentum stresses at saturation, $D_{\mathrm{M}}$, to be

$$
D_{\mathrm{M}}=\frac{m(\bar{u}-c)^{4}}{2 H \delta^{3} N^{3}}\left\{1-\frac{3 H}{(\bar{u}-c)} \frac{\partial \bar{u}}{\partial z}\right\}
$$

where $m$ is the zonal wavenumber; $\bar{u}$ is the zonally averaged wind; $c$ is the phase speed of the gravity wave, $\delta=$ $\left(1+l^{2} / m^{2}\right)^{0.5}, l$ is the meridional wavenumber; and $N$ is the buoyancy frequency $=1.5 \times 10^{-3} \mathrm{sec}^{-2}$. From the dispersion relation the vertical wavenumber $\gamma$ is given by

$$
\gamma=\left[\left(\frac{\delta N}{\bar{u}-c}\right)^{2}-m^{2} \delta^{2}-\frac{1}{4 H^{2}}\right]^{1 / 2}
$$

This yields numerically a vertical wavelength of $\lambda_{z} \sim 200$ $\mathrm{km}$ or $\sim 2 H$ in the thermosphere, for $\bar{u}-c \sim 60 \mathrm{~m} \mathrm{sec}^{-1}$, (an estimate based on Flasar and Conrath, 1990), $H \sim 100$ $\mathrm{km}, m \sim l \sim 2 \pi /(500 \mathrm{~km})$, and $\partial \bar{u} / \partial z \sim 0$. For these values $D_{\mathrm{M}} \sim 1 \times 10^{9} \mathrm{~cm}^{2} \mathrm{sec}^{-1}$ and $K=D_{\mathrm{M}} / P_{\mathrm{r}}$, where $P_{\mathrm{r}}$ is the eddy Prandtl number (Strobel 1989). Thus with an eddy Prandtl number $\sim 2-3$ (cf. Fritts and Dunkerton 1985, Strobel et al. 1985) one obtains the inferred values of $K$ at the homopause $\sim(3-5) \times 10^{8} \mathrm{~cm}^{2} \mathrm{sec}^{-1}$. Note that $\gamma H>1$, so that the gravity waves are hydrostatic and the dispersion relation (8) is valid.

The $\lambda_{z} \sim 1 \mathrm{~km}$ gravity waves derived by Hinson and Tyler (1983) from intensity and frequency fluctuations observed during the Voyager 1 radio occultation of Titan's equatorial troposphere are probably forced in the troposphere, e.g., by convection, in the presence of considerably weaker $\bar{u}-c$ near the surface, perhaps $\sim 1 \mathrm{~m} \mathrm{~s}^{-1}$. These small scale gravity waves, however, cannot propagate upward into strong stratospheric zonal winds aloft (Flasar and Conrath (1990) because $\gamma^{2}$ becomes $<0$ and these waves become evanescent; i.e., they are trapped in the troposphere. Only larger scale gravity waves can propagate to the homopause.

Based on the analytic expressions derived by Strobel et al. (1985) and Strobel (1989), the cooling/heating rate due to gravity wave saturation can be estimated by

$$
H_{\mathrm{gw}}=\frac{N^{2} K_{0}}{2 c_{\mathrm{p}}} e^{\kappa \zeta}\left(P_{\mathrm{r}}-6+7 \kappa\right) .
$$

The heating rate depends very critically on the values of $\kappa$ and $P_{\mathrm{r}}$, for which the latter is poorly constrained by any data in Titan's atmosphere. For homopauses in the range $z=925-1125 \mathrm{~km}$ with $\kappa=0.625$ and $P_{\mathrm{r}} \sim 2$, gravity waves heat the thermosphere by an amount $H_{\mathrm{gw}} \sim 1.5-9 \mathrm{~K} \mathrm{day}^{-1}$ (corresponding respectively to the altitude range 925
$1125 \mathrm{~km}$ ), which is approximately 0.2 times the solar heating rate (Yelle 1991). Although not negligible, gravity wave saturation does not have to dominate the heat balance of the thermosphere even with an eddy coefficient as large as $10^{9} \mathrm{~cm}^{2} \mathrm{sec}^{-1}$ at the homopause, if $P_{\mathrm{r}}>1$. To be more explicit $H_{\mathrm{gw}}=0$, when $\kappa=0.5$ and $P_{\mathrm{r}}=2.5$ and when $\kappa=0.625$ and $P_{\mathrm{r}}=1.5$.

\section{ENERGY SOURCES}

For the level of solar activity at the time of the Voyager 1 encounter with Titan, the appropriately scaled Hinteregger et al. (1981) reference solar EUV fluxes predict a total solar power input to Titan's thermosphere of $3 \times 10^{9}$ $\mathrm{W}$ for wavelengths below $1050 \AA$ and $2 \times 10^{9} \mathrm{~W}$ for wavelengths below $800 \AA$.

Our description of the interaction of magnetospheric plasma with Titan's upper atmosphere is based on the plasma properties summarized by Neubauer $e t$ al. (1984). In particular, the plasma consists of $\mathrm{H}^{+}$, with density = $0.1 \mathrm{~cm}^{-3}$, and $\mathrm{N}^{+}$, with density $=0.2 \mathrm{~cm}^{-3}$, for a total electron density of $0.3 \mathrm{~cm}^{-3}$. The Maxwellian core of the electron distribution function has a characteristic temperature of $200 \mathrm{eV}$ with a suprathermal tail represented by a temperature of $1 \mathrm{keV}$ and a density of $0.01 \mathrm{~cm}^{-3}$. For electron energies greater than $20 \mathrm{keV}$, the energy flux is negligible in comparison to the $\mathrm{keV}$ suprathermal tail (Maclennan et al. 1982) and would penetrate deep into the UV absorbing hydrocarbon region of Titan's upper atmosphere. The measured plasma flow speed was 120 $\mathrm{km} \mathrm{sec}{ }^{-1}$, slower than the corotational velocity, $200 \mathrm{~km}$ $\mathrm{sec}^{-1}$. Titan's orbital velocity is $5.57 \mathrm{~km} \mathrm{sec}^{-1}$. From these quantities an Alfven Mach number of 1.8 and an Alfven conductance of $\sim 4.5$ mho are derived. Based on models for Titan's upper ionosphere (e.g., Ip 1990) the ionospheric Pedersen conductivity was estimated to be $\sim 4500$ mho with a peak value of $\sim 0.01 \mathrm{mho}^{-1}$ near the exobase, $z \sim 1500 \mathrm{~km}$. With the ratio of Alfven conductance to ionospheric conductivity only $\sim 10^{-3}$, direct convection of plasma into the ionosphere is improbable for reasons discussed by Hill et al. (1983). The principal mechanism for entry of energetic particles into Titan's extended atmosphere will be curvature drift toward lower altitudes as the curved magnetospheric flux tubes flow past Titan as argued by Hartle et al. (1982).

The curvature drift speed is $V_{\mathrm{D}}=\left(c m_{\mathrm{e}} v_{\|}^{2}\right) /\left(e R_{\mathrm{Curv}} B\right)$, where $m_{\mathrm{e}}$ is the electron mass, $v_{\|}$is the velocity parallel to the magnetic field $\mathrm{B}, e$ is the electron charge, and $R_{\text {Curv }}$ is the radius of curvature of the draped field lines. The radius of curvature is assumed equal to the radius of the ionopause, $R_{\mathrm{i}}$. The inward drift per pass through the exosphere is $\delta=V_{\mathrm{D}}\left(\pi R_{\mathrm{Curv}} / v_{\|}\right) \sim\left(c m_{\mathrm{e}} v_{\|} \pi\right) /(e B)$. The ionopause is defined for our purposes as the altitude where the drift time of flux tubes slowed down by mass loading is 
comparable to the time constant for frictional drag $\tau_{f} \sim$ $\nu_{\text {in }}^{-1}$, where $\nu_{\text {in }}$ is the ion-neutral collision frequency. From the inferred density profile in Titan's upper atmosphere, the ionopause is located at $R_{\mathrm{i}} \sim 4400 \mathrm{~km}$, a result identical to Hartle et al. (1982). The total drift distance $d=2 \delta \tau_{\mathrm{D}} / \tau_{\mathrm{B}}$, where $\tau_{\mathrm{D}}$ is the drift time of a flux tube through the atmosphere $(\sim 900 \mathrm{sec})$ and $\tau_{\mathrm{B}}$ is the bounce period $(\sim 70-500 \mathrm{sec}$ for electron energies in the $0.2-10 \mathrm{keV}$ range). Curvature drift speeds vary from $\sim 20 \mathrm{~km} \mathrm{sec}^{-1}$ at $0.2 \mathrm{keV}$ to $\sim 900 \mathrm{~km}$ $\mathrm{sec}^{-1}$ at $10 \mathrm{keV}$. Over the same energy range the inward drift per pass varies from 30 to $200 \mathrm{~km}$, whereas the total drift distance increases from 180 to $8700 \mathrm{~km}$.

Based on derivations presented in Strobel et al. (1990) for Triton's magnetosphere-ionosphere interaction, the power, $P_{\mathrm{m}}$, delivered to Titan's ionopause is given by the expression

$$
P_{\mathrm{M}}=1.6 \times 10^{-6} \pi R_{\mathrm{i}} \int_{0.1}^{10} 4 \pi \Phi(E) E \delta(E) d E,
$$

where $\Phi(E)$ is the measured electron flux in units of $\mathrm{cm}^{-2}$ $\mathrm{sec}^{-1} \mathrm{sr}^{-1} \mathrm{keV}^{-1}$, the energy $E$ is in $\mathrm{keV}, \delta(E)$ is in $\mathrm{km}$, and $R_{\mathrm{i}}$ is in $\mathrm{km}$. For the adopted plasma properties this expression yields an input magnetospheric power of $2.3 \times 10^{8} \mathrm{~W}$ to the hemisphere into which the electrons drift (the direction given by the vector $-\mathbf{R}_{\text {curv }} \times \mathbf{B}$ ). At the time of the Voyager 1 flyby of Titan this hemisphere essentially coincided with the sunlit hemisphere. The magnetospheric power is $\sim 7 \times 10^{7} \mathrm{~W}$ in the thermal range $(<0.7 \mathrm{keV})$ and $\sim 1.6 \times 10^{8} \mathrm{~W}$ in the suprathermal range $(0.7-10 \mathrm{keV})$. To obtain a much larger magnetospheric power dissipation rate would require depletion of the energy content of all flux tubes which intersect Titan's extended atmosphere by direct convective flow. Then $\boldsymbol{P}_{\mathrm{M}}$ $5 \times 10^{9} \mathrm{~W}$ could be achieved, but we consider this scenario extremely improbable.

\section{CALCUlation OF ULTRAVIOLET EMISSIONS}

Atmospheric excitation rate calculations by direct absorption of solar photons and indirectly by photoelectrons were performed with the photoelectron model of Strickland and Meier (1982) updated with more recent cross sections as described in Strickland et al. (1989). Magnetospheric electron transport and energy deposition on Titan were modeled with the auroral code developed by Strickland et al. (1989) for the Earth with the assumptions of a pure $\mathrm{N}_{2}$ atmosphere and tangential penetration as discussed by Strobel et al. (1990) in the context of magnetospheric-ionospheric interaction on Triton. Updated cross sections for $\mathrm{N}_{2}$ emission rates were adopted from Ajello et al. (1989) and James et al.(1990). The emission rates for the $\mathrm{N}^{+}$multiplets at 916 and $1085 \AA$ by photodissociative ionization via the dissociative $\mathrm{H}$ state of $\mathrm{N}_{2}^{+}$were calcu- lated with yields of 0.042 and 0.184 based on the laboratory and observational analyses of Meier et al. (1991). These calculations were performed as a function of $\mathrm{N}_{2}$ column density and transformed to the model atmosphere shown in Fig. 6. Although the total inelastic energy loss for electrons and EUV photon flux attenuation $(\lambda<800$ $\AA$ ) were computed as if the atmosphere were pure $N_{2}$, the resulting atmospheric excitation rates calculated for a pure $\mathrm{N}_{2}$ atmosphere were then multiplied by the tropopause $\mathrm{N}_{2}$ mixing ratio $\left(=1-f_{\mathrm{CH}_{4}}-f_{\mathrm{Ar}}\right.$ evaluated at the tropopause) to obtain the appropriate excitation rates in a three-constituent atmosphere. Solar radiation was assumed incident at $60^{\circ}$ zenith angle to model airglow emission above the absorbing $\mathrm{CH}_{4}$ region, whereas magnetospheric power was assumed to be dissipated uniformly over the hemisphere into which electrons were drifting which as discussed above coincided with the sunlit hemisphere.

Three model atmospheres were considered for the ultraviolet emission intensity calculations: (1) a pure $\mathrm{N}_{2}$ atmosphere for reference purposes, (2) the $2.6 \% \mathrm{CH}_{4}$ model atmosphere representing the lower limit on $\mathrm{CH}_{4}$ abundance to obtain a good fit to the Voyager UVS solar occultation data, and (3) the $3.4 \% \mathrm{CH}_{4}$ model atmosphere representing the upper limit on $\mathrm{CH}_{4}$ abundance from IRIS data. The $5 \% \mathrm{CH}_{4}$ model atmosphere was not considered because it violated the IRIS constraint.

\section{AIRGLOW RESULTS}

From our calculations of a large number of $\mathrm{N}_{2}, \mathrm{~N}$, and $\mathrm{N}^{+} \mathrm{UV}$ airglow excitation rates the intensities of the following emission features were selected for discussion: $\mathrm{N}_{2} c_{4}^{\prime \prime} \sum_{u l}^{+}$Rydberg (0-0) and (0-1) bands, the $\mathrm{N}^{+}$multiplets at 916 and $1085 \AA$, and the $\mathrm{N}_{2} \mathrm{LBH}$ bands. The $\mathrm{N}_{2}$ LBH bands are entirely due to electron impact and are essentially optically thin, with only weak absorption by $\mathrm{CH}_{4}$. The $\mathrm{N}^{+}$multiplets are also optically thin and only the 916- $\AA$ multiplet is subject to significant absorption by $\mathrm{N}_{2}$, because the 916.701- and 916.710- $\AA$ lines of the $\mathrm{N}^{+}$ sextuplet (55\% of the intensity) coincide with rotational lines of the $\mathrm{N}_{2} b^{\prime} \Pi_{u}(11-0)$ band $\left[J^{\prime}-J^{\prime \prime}=(4-5),(6-6)\right.$, $(8-7)]$ and are absorbed with an effective cross section $\sim 2.9 \times 10^{-17} \mathrm{~cm}^{2}$ at $180 \mathrm{~K}$ (Meier et al. 1991). The $\mathrm{N}_{2}$ $c_{4}^{\prime}(0-0)$ and $(0-1)$ bands were chosen because they are the most prominent features of Titan's EUV emission. The strongest rotational line in the $c_{4}^{\prime}(0-0)$ band reaches unit optical depth at line center when the $\mathrm{N}_{2}$ column density is only $\sim 3 \times 10^{14} \mathrm{~cm}^{-2}$ (Ajello et al. 1989). Also, the emission branching ratio for the $(0-1)$ band is 0.14 from $c_{4}^{\prime \prime} \Sigma_{u}^{+}\left(v^{\prime}=0\right)$ state. Thus a detailed interpretation of these $c_{4}^{\prime}$ bands requires a radiative transfer analysis with optical thickness effects, predissociation, branching, etc., included, which is beyond the scope of this paper. An 
TABLE II

Sources of Titan Nitrogen Airglow

\begin{tabular}{|c|c|c|c|c|c|}
\hline \multirow[t]{2}{*}{ Processes } & \multicolumn{5}{|c|}{ Disk Center Emission Intensities ${ }^{1}$} \\
\hline & $N^{+}(916 \dot{A})^{2,3}$ & $\begin{array}{c}\mathrm{N}_{2}(958 \dot{\mathrm{A}}) \\
\mathrm{c}_{4}^{\prime}(0-0)\end{array}$ & $\begin{array}{c}N_{2}(981 \dot{A}) \\
c_{4}^{\prime}(0-1)\end{array}$ & $\mathrm{N}^{+}(1085 \dot{\mathrm{A}})^{3}$ & $\mathrm{~N}_{2}$ LBH \\
\hline Observed(revised calibration) & $0.6 \mathrm{R}$ & $8 \mathbf{R}$ & $6 \mathrm{R}$ & $8 \mathbf{R}$ & $96 \mathrm{R}$ \\
\hline \multicolumn{6}{|l|}{ Model $0 \% \mathrm{CH}_{4}$} \\
\hline$\cdot \mathrm{N}_{2}+\mathrm{h} v \rightarrow \mathrm{N}_{2}+(\mathrm{H})$ & $2.0 \mathrm{R}$ & & & $8.4 \mathrm{R}$ & \\
\hline - photoelectrons $+\mathrm{N}_{2}$ & $0.2 \mathrm{R}$ & $9.0 \mathrm{R}$ & $1.5 \mathrm{R}$ & $2.2 \mathrm{R}$ & $94 \mathrm{R}$ \\
\hline -magnetospheric $\mathrm{C}^{4}+\mathrm{N}_{2}$ & $\underline{0.1 R^{5}}$ & $2.0 \mathrm{R}^{5}$ & $\underline{0.3 R^{5}}$ & $\underline{0.8 R^{5}}$ & $8.6 \mathrm{R}$ \\
\hline Total & $2.3 \mathrm{R}$ & $11 \mathrm{R}$ & $1.8 \mathrm{R}$ & $11 \mathrm{R}$ & $103 \mathrm{R}$ \\
\hline \multicolumn{6}{|l|}{ Model $2.6 \% \mathrm{CH}_{4}$} \\
\hline$-\mathrm{N}_{2}+\mathbf{h} v \rightarrow \mathrm{N}_{2}{ }^{+}(\mathrm{H})$ & $1.5 \mathrm{R}$ & & & $7.0 \mathrm{R}$ & \\
\hline$\cdot$ photoelectrons $+\mathrm{N}_{2}$ & $0.04 \mathrm{R}$ & $5.8 \mathrm{R}$ & $0.96 \mathrm{R}$ & $1.2 \mathrm{R}$ & $92 \mathrm{R}$ \\
\hline - magnetospheric $\mathrm{c}^{4}+\mathrm{N}_{2}$ & $\underline{0.09 R^{5}}$ & $1.5 \mathrm{R}^{5}$ & $\underline{0.3 R^{5}}$ & $\underline{0.6 R^{5}}$ & $\underline{8.2 \mathrm{R}}$ \\
\hline Total & $1.6 \mathrm{R}$ & $7.3 \mathbf{R}$ & $1.3 \mathrm{R}$ & $8.8 \mathrm{R}$ & $100 \mathrm{R}$ \\
\hline \multicolumn{6}{|l|}{ Model 3.4\% $\mathrm{CH}_{4}$} \\
\hline$\cdot \mathrm{N}_{2}+\mathrm{h} v \rightarrow \mathrm{N}_{2}{ }^{+}(\mathrm{H})$ & $1.3 \mathrm{R}$ & & & $6.0 \mathrm{R}$ & \\
\hline$\cdot$ photoelectrons $+\mathrm{N}_{2}$ & $0.02 \mathrm{R}$ & $5.0 \mathrm{R}$ & $0.8 \mathrm{R}$ & $1.0 \mathrm{R}$ & $78 \mathrm{R}$ \\
\hline - magnetospheric $\mathrm{e}^{-}+\mathrm{N}_{2}$ & $\underline{0.08 R^{5}}$ & $\underline{1.4 R^{5}}$ & $\underline{0.2 \mathrm{R}^{5}}$ & $\underline{0.5 \mathrm{R}^{5}}$ & $7.0 \mathrm{R}$ \\
\hline Total & $1.4 \mathrm{R}$ & $6.4 \mathrm{R}$ & $1.0 \mathrm{R}$ & $7.5 \mathrm{R}$ & $85 \mathrm{R}$ \\
\hline
\end{tabular}

\footnotetext{
${ }^{1}$ Observations are slit-averaged intensities.

${ }^{2}$ Includes $\mathrm{N}_{2}$ absorption.

${ }^{3}$ Meier et al.(1991) with yields $\phi_{916}=0.042, \phi_{1085}=0.184$.

${ }^{4}$ For power $=2.3 \times 10^{8} \mathrm{~W}$.

${ }^{5}$ Includes $\mathrm{CH}_{4}$ absorption.
}

earlier, initial attempt by Conway (1983) indicated that strong mutual perturbations of the $c_{4}^{\prime}$ and $b^{\prime}$ states argued against detailed self-absorption calculations for band systems originating from these states.

\section{5a. Disk Center Results}

In Table II center-of-disk averaged calculations for the three model atmospheres are reported by specific process: solar excitation of the $\mathrm{N}_{2}^{+}(\mathrm{H})$ state, photoelectron excitation, and excitation by precipitation of magnetospheric electrons and compared with the intensities given by Strobel et al. (1991) which are downward revisions of the original Strobel and Shemansky (1982) intensities. The absolute intensities from a reanalysis of the Voyager Titan EUV data by Hall et al. (1992) were not in final form to be used in this study. It should be kept in mind that the "disk-averaged" intensities reported by Strobel and Shemansky (1982) were not really disk averaged but averaged over the UVS slit. The slit averaged intensities should be bounded by $(0.67-1)$ times the calculated disk center intensities.

From Table II one concludes that a pure $\mathrm{N}_{2}$ atmosphere yields intensities consistent with the observations in the limit of subsolar, center of the disk values. No formal error bars were put on the data by Strobel and Shemansky (1982) but in light of the revised calibration by Holberg $e t$ al. (1982, 1991), signal-to-noise, $30-\AA$ spectral resolution, etc., error bars on bright spectral features of $\pm 50 \%$ would not seem unreasonable. Given the low spectral resolution of the Voyager UVS, the inferred intensity of a weak narrow feature such as the $\mathrm{N}^{+}(916 \AA)$ multiplet is very uncertain and not much significance should be put on the differences between observations and calculations. 

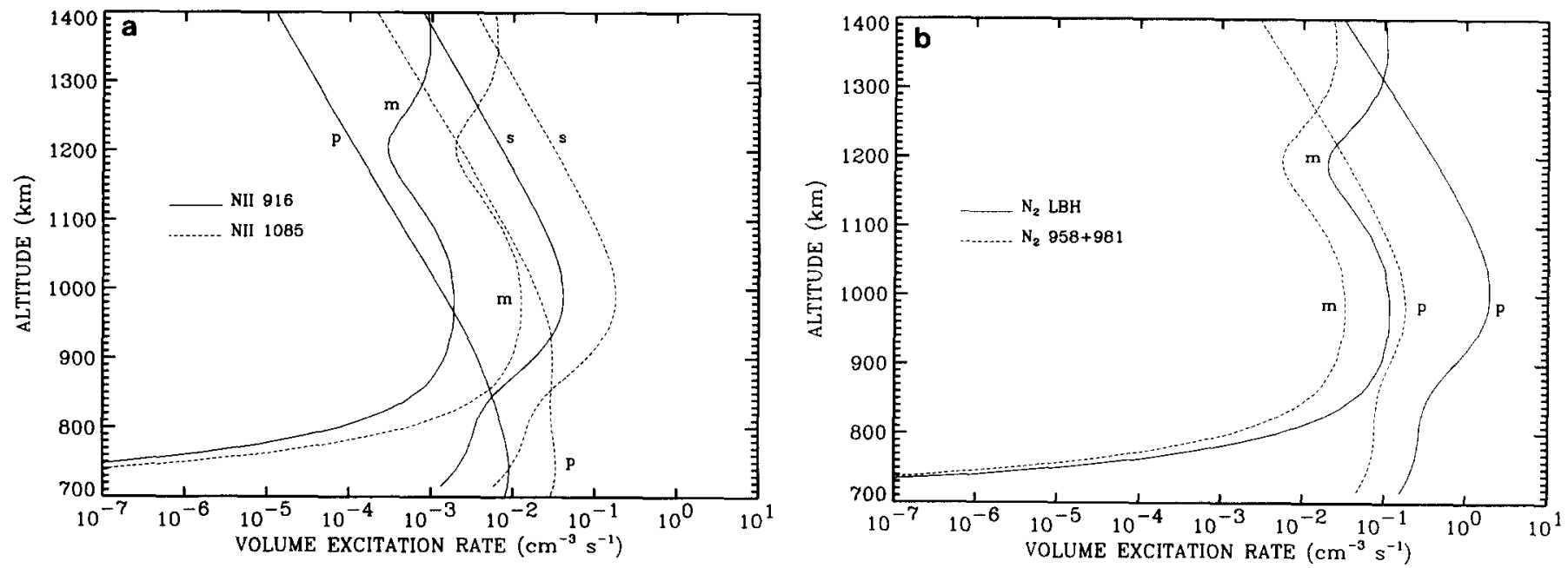

FIG. 8. Volume excitation rates in cubic centimeters per second as a function of altitude: (1) due to solar excitation via $\mathrm{N}_{2}^{+}(\mathrm{H})$ state $(s)$, (2) due to photoelectrons $(p)$, and (3) due to magnetospheric electrons $(m)$ for a pure $\mathrm{N}_{2}$ atmosphere. (a) $\mathrm{N}^{-}$multiplets and (b) $\mathrm{N}_{2}$ bands.

But the UVs solar occultation data requires $f_{\mathrm{CH}_{4}} \sim 0.08$ at $1125 \mathrm{~km}$ and the impact of $\mathrm{CH}_{4}$ on the emergent intensities is illustrated by the 2.6 and $3.4 \%$ model atmospheres in Table II. For emission features shortward of Ly $\alpha$ there is a $22-33 \%$ reduction in the calculated intensities for the $2.6 \%$ model and a $35-45 \%$ reduction for the $3.4 \%$ model. Even the LBH bands suffer a $17 \%$ decrease in intensity for the latter model. The intensity reductions result from the fractional decrease in the $\mathrm{N}_{2}$ content of the atmosphere and absorption by $\mathrm{CH}_{4}$. The $\mathrm{N}_{2} c_{4}^{\prime}(0-0)$ and $(0-1)$ band intensities predicted for these models are too low when compared with observations, whereas the $\mathrm{N}^{+}(1085 \AA)$ and $\mathrm{N}_{2}$ LBH bands are consistent with observations. According to Hall et al. (1992) the $\mathrm{N}_{2} \mathrm{LBH}$ band intensities given by Strobel and Shemansky (1982) should be regarded as tentative and not given much weight.

\section{5b. Volume Excitation Rates}

To understand the contribution of $\mathrm{CH}_{4}$ absorption to the disk center intensities the volume excitation rate profiles for the various emissions are instructive. The solar excitation of the $\mathrm{N}_{2}^{+}(\mathrm{H})$ state peaks at $-975 \mathrm{~km}$ (Fig. 8a) and is the major source of $\mathrm{N}^{+}(1085-$ and $916-\AA)$ multiplets as is evident from Table II. Photoelectron excitation of the $\mathrm{LBH}$ bands peaks at $\sim 1000 \mathrm{~km}$, but excitation of $\mathrm{N}^{+}(916-\AA)$ multiplet maximizes at $\sim 725 \mathrm{~km}$ (Fig. 8). The latter excitation is dominated by soft $\mathrm{X}$ rays because dissociative ionization of $\mathrm{N}_{2}$ and excitation of $\mathrm{N}^{+}$requires a threshold energy of $38 \mathrm{eV}$. Thus only the most energetic solar photons are capable of producing photoelectrons in excess of $38 \mathrm{eV}$ and these photons are absorbed by $\mathrm{N}_{2}$ with a significantly smaller cross section than the photons responsible for excitation of the $\mathrm{N}_{2}^{+}(\mathrm{H})$ state and the photoelectrons that generate most of the LBH emission, whose upper state has a threshold of only $8.5 \mathrm{eV}$. Electron impact excitation of the $\mathrm{N}_{2} c_{4}^{\prime}$ system and of $\mathrm{N}^{+}(1085 \AA)$ from $\mathrm{N}_{2}$ have respective thresholds of $\sim 13$ and $36 \mathrm{eV}$ and thus are intermediate between the profiles for LBH and $\mathrm{N}^{+}(1085 \AA)$. Excitation of these emission features by magnetospheric electrons is characterized by a peak at $\sim 1350$ $\mathrm{km}$ due to the Maxwellian core of the electron distribution function, with $T_{\mathrm{e}}=200 \mathrm{eV}$ and a lower peak at $\sim 975 \mathrm{~km}$ due to the suprathermal tail of its distribution, with $T_{\mathrm{e}} \sim$ $1 \mathrm{keV}$ as shown in Fig. 8. Photons emitted upward from the high altitude peak will not be absorbed by $\mathrm{CH}_{4}$, but photons emitted from the lower peak encounter a vertical $\mathrm{CH}_{4}$ column density of $\sim 1 \times 10^{16} \mathrm{~cm}^{-2}$.

\section{5c. Bright Limb Results}

From the volume excitation rates presented in the previous section, it is straightforward to calculate bright limb profiles for three model atmospheres produced by the Sun at a $60^{\circ}$ zenith angle and viewed by a spacecraft at a $90^{\circ}$ angle to the Sun-Titan line. This geometry was selected to approximate the finite width of the UVS slit projected on Titan's atmosphere $(\sim 200 \mathrm{~km})$ and the bias of preferential collection of photons emitted from the closer half of the sunlit hemisphere due to $\mathrm{CH}_{4}$ absorption. Magnetospheric excitation was uniformly distributed over the sunlit hemisphere.

In Fig. 9 bright limb profiles are shown for direct solar plus photoelectron excitation in a pure $\mathrm{N}_{2}$ atmosphere. Note that the limb profiles maximize at $\sim 925 \mathrm{~km}$, with the $\mathrm{N}_{2} c_{4}^{\prime}$ bands exhibiting a secondary maximum/ledge at $\sim 750 \mathrm{~km}$ due to soft $X$ rays. The equivalent limb profiles due to magnetospheric electron excitation are given in Fig. 9. The distinct signatures of thermal and suprathermal electrons are evident at 1300 and $900 \mathrm{~km}$, respectively. 


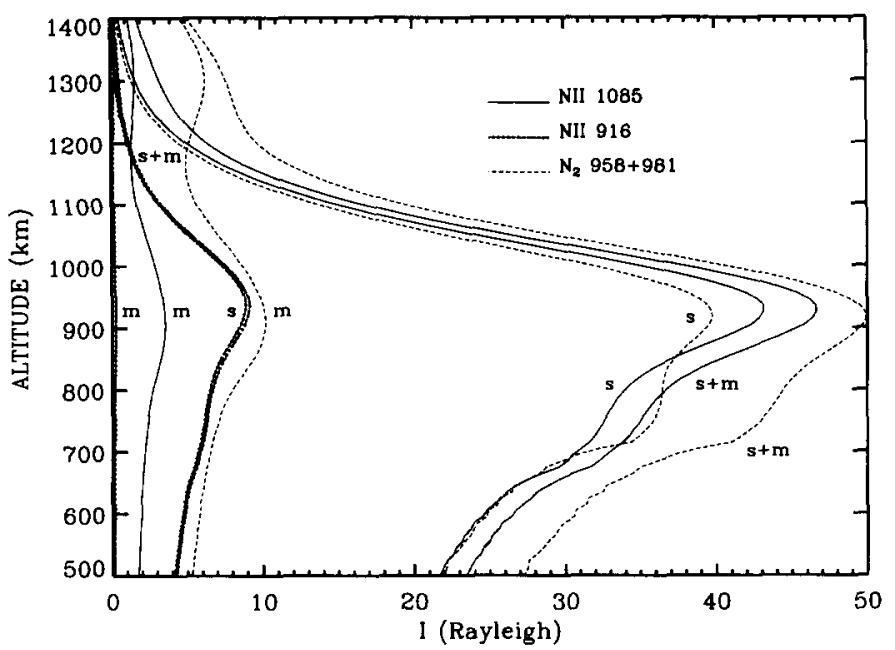

FIG. 9. Calculated bright-limb profiles as a function of altitude (1) due to solar/photoelectron excitation $(s),(2)$ due to magnetospheric electron excitation $(m)$, and (3) the sum of solar/photoelectron and magnetospheric electron excitation for a pure $\mathrm{N}_{2}$ atmosphere $(s+m)$.

The combined limb profiles from these two sources of excitation are presented in Fig. 9 and may be compared to the revised peak intensities based on Strobel and Shemansky (1982) of 2,25 , and $25 R$ for $\mathrm{N}^{+}(916 \AA), \mathrm{N}_{2}$ $c_{4}^{\prime}(0-0,958-\AA)$, plus $(0-1,981-\AA)$ bands, and $\mathrm{N}^{+}(1085 \AA)$, respectively. It is evident from Fig. 9 that our calculations could easily account for the revised intensities and even the originally reported intensities, if predissociation can be neglected in the $\mathrm{N}_{2} c_{4}^{\prime}$ system and Titan's atmosphere were pure $\mathrm{N}_{2}$.

The rather dramatic effect of $\mathrm{CH}_{4}$ absorption can be seen by a comparison of Fig. 10a for the $2.6 \% \mathrm{CH}_{4}$ model atmosphere and Fig. 11 for the $3.4 \% \mathrm{CH}_{4}$ model atmosphere with Fig. 9. From Figs. 10 and 11 it is noted that $\mathrm{CH}_{4}$ absorption eliminates the soft $\mathrm{X}$-ray excitation signature evident in a pure $\mathrm{N}_{2}$ atmosphere at $\sim 750 \mathrm{~km}$ and reduces peak intensities by factors of 3-5. Also the peak intensities are produced $\sim 50-100 \mathrm{~km}$ higher in altitude due to $\mathrm{CH}_{4}$ absorption. An even more significant effect is evident in the limb profiles due to magnetospheric electron excitation where the dominant contribution of suprathermal electrons at $900 \mathrm{~km}$ (Fig. 9) is reduced to a weak ledge in the profiles of Figs. 10 and 11 at $\sim 1150 \mathrm{~km}$. Thermal electrons generate peaks at $1300 \mathrm{~km}$ (Figs. 10 and 11) which are comparable in intensity to those calculated for the pure $\mathrm{N}_{2}$ atmosphere because the $\mathrm{CH}_{4}$ model atmospheres are optically thin at this high altitude. The combined limb profiles from these two sources of excitation are presented in Figs. 10 and 11. When compared with Fig. 9, it is evident that $\mathrm{CH}_{4}$ absorption reduces peak intensities by factors of $2-5$ and displaces the predicted peaks upward by $75-100 \mathrm{~km}$. If predissociation effects were taken into consideration, the predicted $\mathrm{N}_{2} c_{4}^{\prime}(0-0$, $958 \AA)$ and $(0-1,981 \AA)$ band intensities would be below the lower limits on the observed intensities, whereas the $\mathrm{N}^{+}(1085-\AA)$ intensities are within the observational error bars. Although the predicted $\mathrm{N}^{+}(916-\AA)$ intensities are in excellent agreement with observations, we don't regard this weak feature as a benchmark.

A further comparison of Figs. 9, 10a, and 11 indicates that the $\mathrm{N}_{2} c_{4}^{\prime}(0-0,958-\AA)$ and $(0-1,981-\AA)$ band limb profiles contain two important signatures in their profile shapes. The ratio of peak intensity to the ledge intensity in the 700- to $800-\mathrm{km}$ region (cf. Fig. 9) is indicative of the $\mathrm{CH}_{4}$ absorption and hence its abundance in the upper atmosphere. Whereas the ratio of the peak intensity to the ledge intensity in the $1200-$ to $1300-\mathrm{km}$ region is a measure of the ratio of excitation rates by photoelectrons to magnetospheric thermal electrons. To illustrate this latter point limb profile calculations were performed with the magnetospheric power dissipation increased by a factor of 3 for the $2.6 \% \mathrm{CH}_{4}$ model atmosphere. The profile is shown in Fig. 10b. Note that magnetospheric electrons produce a secondary maximum at $1300 \mathrm{~km}$ with intensity $\sim 90 \%$ of the peak intensity at $1000 \mathrm{~km}$. This shape is inconsistent with UVS observations and constrains the inferred magnetospheric power dissipation to be close to our estimates in Section 3.

Because this point is very important it is necessary to degrade the spatial resolution of the calculated limb profile by the finite UVS slit width $(\sim 200 \mathrm{~km})$ projected on the limb with a computer program generously supplied by D. T. Hall and then to compare this with the observed limb profile illustrated in Fig. 2 of Hall et al. (1992). One ambiguity in this comparison is the actual location of peak bright limb emission to the apparent observed emission. Strobel and Shemansky (1982) reported the observed bright limb intensity peak for $\mathrm{N}_{2}$ emission (942-998 $\AA$ ) at $z \sim 1085 \mathrm{~km}$. Conway (1983) argued that the $\mathrm{N}_{2} c_{4}^{\prime}$ bands should behave similarly to the $\mathrm{N}_{2} \mathrm{BH}$ bands that he modeled for Titan's upper atmosphere. According to Conway's calculations the limb brightening reported by Strobel and Shemansky (1982) for the $\mathrm{N}_{2} c_{4}^{\prime}(0-0)$ band implies an excitation source at $z \geq 1400 \mathrm{~km}$ in contradiction to the reported altitude peak of $z \sim 1085 \mathrm{~km}$. Another attempt to locate the peak limb emission indicated that it occurred at $z \sim 1325 \mathrm{~km}$ (Hunten et al. 1984). This determination of the bright limb peak supported the conclusions of Conway (1983).

But the most recent JPL determination, at our request, yielded peak emission at $z=765 \pm 50 \mathrm{~km}$ based on Voyager optical navigation and NAIF (C. H. Acton and T.-C. M. Wang 1991, private communication). We clearly do not have an incontrovertible determination of the peak emission altitude. For the purposes of comparison we adjusted the UVS data upward by $\sim 300 \mathrm{~km}$ to obtain peak 

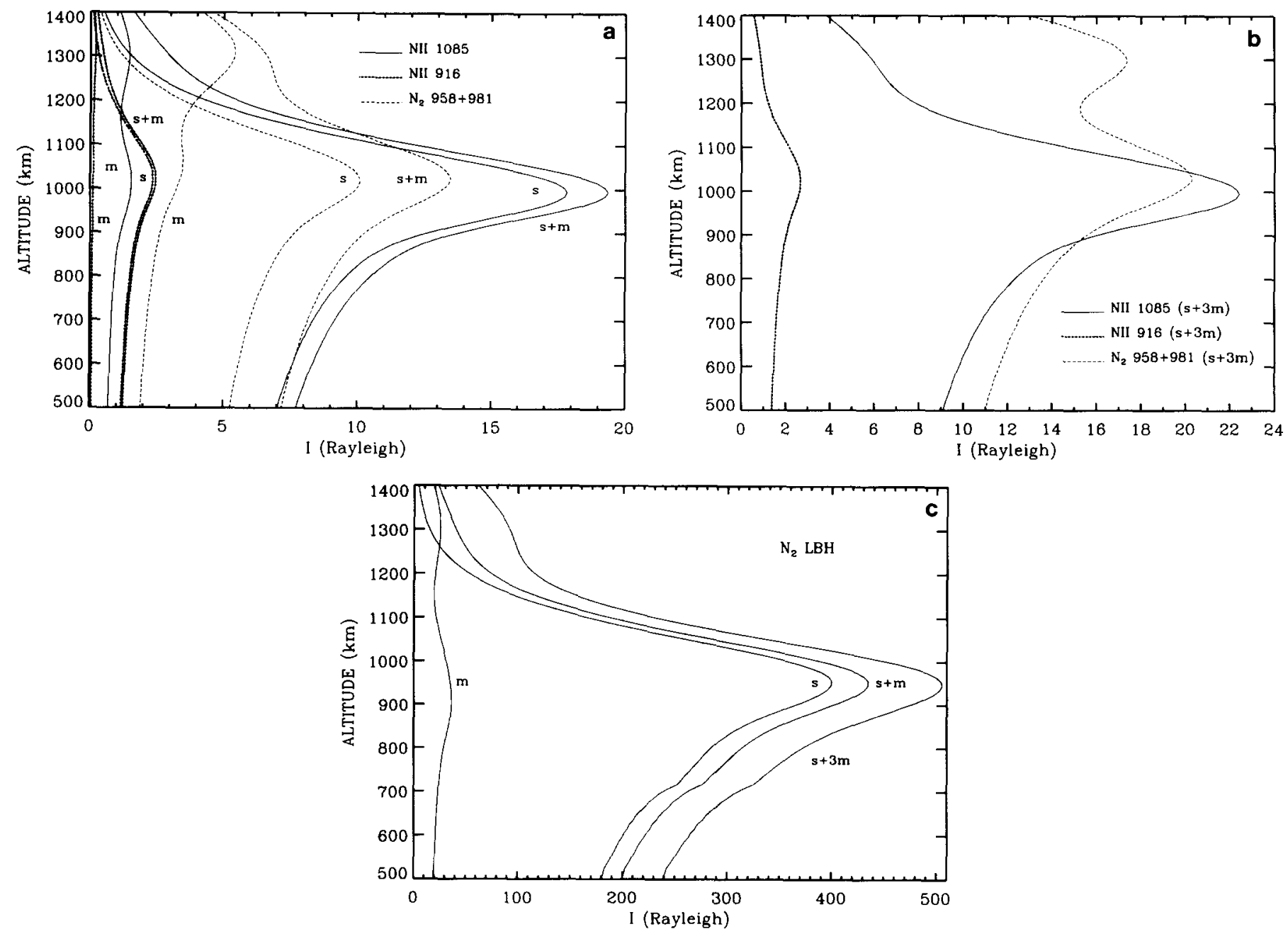

FIG. 10. Same as Fig. 9, but for the $2.6 \% \mathrm{CH}_{4}, 1.4 \%$ Ar model atmosphere. $\mathrm{b}$ is the same as a but with three times the magnetospheric electron excitation rates $(s+3 m)$. c also illustrates bright-limb profiles for the $\mathrm{N}_{2} \mathrm{LBH}$ bands by solar/photoelectron excitation $(s)$, magnetospheric electron excitation $(m)$, the sum of $s+m$, and the sum of $s+3 m$.

emission at $1050 \mathrm{~km}$ which coincides with the calculated peaks (Fig. 12). The only calculated bright limb profile which would be consistent with the observed peak emission at $z=765 \pm 50 \mathrm{~km}$ would be our pure $\mathrm{N}_{2}$ atmosphere result (Fig. 9) that has an apparent emission peak at $\sim 800$ $\mathrm{km}$. But this model atmosphere is totally inconsistent with the UVS solar occultation data. In Fig. 12 actual and slitaveraged bright limb profiles for the $2.6 \% \mathrm{CH}_{4}$ model atmosphere with one $[s+m]$ and three times $[s+3 m]$ the magnetospheric excitation rate are compared with the adjusted data. It is evident that the topside $[s+3 m]$ profiles are inconsistent with the observed rapid intensity falloff with altitude. It is apparent that the data exhibit altitude variations faster than the nominal $200-\mathrm{km}$ slit smoothing can generate and for this reason unsmoothed profiles are also compared with data. The conclusion is the same: magnetospheric power dissipation is at most the value obtained from Eq. (10), $2.3 \times 10^{8} \mathrm{~W}$.
At this point one might wonder whether the altitude scale on the UVS solar occultation data and consequently Figs. 1-5 could be in error by values comparable to the uncertainty in the location of the bright limb peak emission. The UVS occultation data distance scale is inherently much more accurate because of the near coincidence of earth occultation by the radio science experiment coupled with a highly accurate ephemeris for Titan and Voyager 1 spacecraft velocity during occultation to translate time into distance. This is demonstrated by the extremely smooth fits of the $\mathrm{N}_{2}$ density and temperature profiles at $900 \mathrm{~km}$ in Fig. 6. Any atmosphere derived from the UVS solar occultation data must smoothly join the atmosphere below derived from IRIS and radio occultation data and extended upward from $\sim 300 \mathrm{~km}$ by the Yelle (1991) radiative transfer model to $900 \mathrm{~km}$.

To complete this section on limb profiles the calculated $\mathrm{N}_{2} \mathrm{LBH}$ bands' limb profiles are presented in Fig. 10c for 


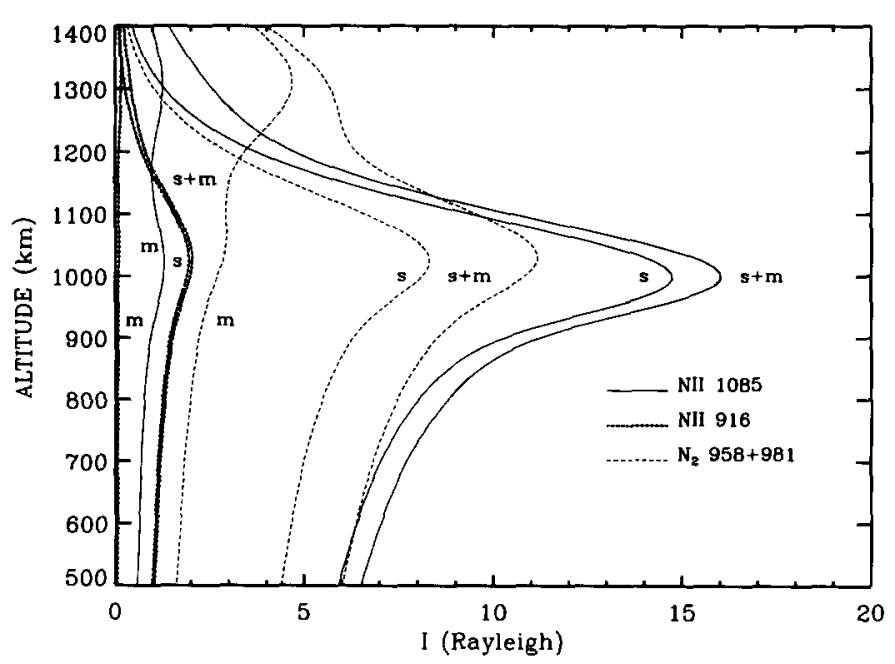

FIG. 11. Same as Fig. 10a, but for the $3.4 \% \mathrm{CH}_{4}, 13.6 \% \mathrm{Ar}$ model atmosphere

the $2.6 \% \mathrm{CH}_{4}$ model atmosphere. Because the $\mathrm{LBH}$ bands are only weakly absorbed by $\mathrm{CH}_{4}$, the peak $\mathrm{LBH}$ intensity at $\sim 925 \mathrm{~km}$ of $430 R$ for photoelectron plus magnetospheric electron excitation differs by less than $10 \%$ from the pure $\mathrm{N}_{2}$ atmosphere calculation. Note that there is only about a 30- $R$ contribution from magnetospheric electrons. Thus the LBH limb profile intensity and also its shape is not diagnostic of magnetospheric power input to Titan's upper atmosphere.

\section{UPPER LIMIT ON ARGON MIXING RATIO}

The $2.6 \%$ and $3.4 \% \mathrm{CH}_{4}$ model atmospheres contain $1.4 \%$ and $13.6 \%$ Ar mixing ratios, respectively. The cross sections for electron impact excitation of the argon resonance lines at 1048 and $1067 \AA$ are large, peaking at (3-4) $\times 10^{-17} \mathrm{~cm}^{2}$ with thresholds $\sim 12 \mathrm{eV}$ (Ajello et al. 1990). The nominal spectral resolution of the Voyager UVS is $30 \AA$ and these two resonance lines of Ar would be observed by the UVS as a single, extremely Dopplerbroadened line rather than as two distinct lines. Given the concerns about absolute calibration of the Voyager UV spectrometers (cf. Holberg et al. 1982) and the underprediction of absolute intensities in comparison with observations in this study, the best method to infer upper limits on the Ar mixing ratio from the nondetection of its resonance lines is by comparison with a close spectral feature of nitrogen. The ideally suited spectral feature is the $\mathrm{N}^{+}(1085-\AA)$ multiplet which is within $40 \AA$ of the $\mathrm{Ar}$ resonance lines and is almost entirely due to solar/photoelectron excitation of $\mathrm{N}_{2}$.

In Table III the disk center emission rates of the argon resonance lines are compared with the $\mathrm{N}^{+}(1085-\AA)$ multiplet for the two model atmospheres. Although these $\mathrm{Ar}$ lines are very optically thick, the emission rates corrected for $\mathrm{CH}_{4}$ absorption are almost identically equal to the integrated excitation rates above the $\tau=1$ level for $\mathrm{CH}_{4}$ absorption. According to R. R. Meier (private communication, 1992), the latter rate should approximate the intensity obtained from a full radiative transfer calculation. Note that photoelectrons account for $85 \%$ of the total excitation of these Ar lines with only a $15 \%$ contribution from magnetospheric electrons. Thus an intensity ratio comparison (Ar lines $/ \mathrm{N}^{+} 1085 \AA$ ) is to the first-order independent of the absolute solar fluxes and the magnetospheric power dissipation and is essentially a function only of the $\mathrm{Ar} / \mathrm{N}_{2}$ mixing ratio. From the first two models in Table III it appears that the disk center intensities of the Ar 1048- and 1067- $\AA$ lines are linearly proportional to the Ar mixing ratio, i.e., a factor of 10 increase in the mixing ratio yields a factor of 10 increase in intensity, although this is probably a result of the limited parameter space explored.

From the upper limit reported by Strobel and Shemansky (1982) $(<6 R$ revised to $4 R$ ) for the $\operatorname{Ar} 1048 \AA$, an upper limit intensity ratio of 0.5 for $\operatorname{Ar} 1048 / \mathrm{N}^{+} 1085$ is derived. The predicted intensity ratio is 0.4 for the $13.6 \%$ Ar model atmosphere and when the Ar I 1067- $\AA$ line intensity is added to the 1048 line intensity this intensity ratio increases to 0.65 . From the results in Table III an Ar mixing ratio in excess of 0.2 can be definitely excluded, because the Ar resonance lines would be as bright as the $\mathrm{N}^{+}(1085-\AA)$ multiplet which is not seen by visual inspection of Fig. 1 in Strobel and Schemansky (1982). We recommend a more stringent Ar upper limit of 0.14 based on our results for the $13.6 \%$ Ar model atmosphere

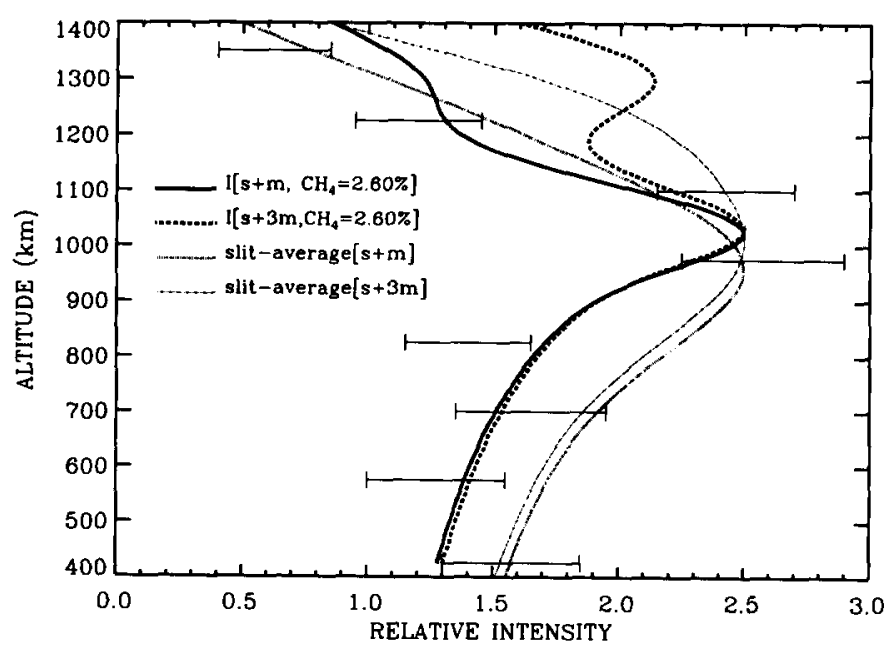

FIG. 12. Comparison of calculated bright limb profiles for the $2.6 \%$ $\mathrm{CH}_{4}$ model atmosphere from Figs. 10a and 10b, cases: $s+m$ and $s+$ $3 m$, unsmoothed and slit-averaged, with UVS observed limb data for $\mathrm{N}_{2}$

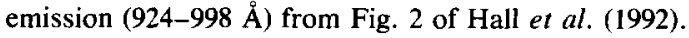


TABLE III

Sources of Titan Argon Airglow

\begin{tabular}{|c|c|c|c|}
\hline \multirow[t]{2}{*}{ Processes } & \multicolumn{3}{|c|}{ Disk Center Emission Intensities ${ }^{1}$} \\
\hline & $\operatorname{Ar}(1048 \dot{A})$ & $\operatorname{Ar}(1048+1067 \dot{A})$ & $N^{+}(1085 \dot{A})^{2}$ \\
\hline Observed(revised calibration) & $<4 \mathrm{R}$ & $<4 \mathrm{R}$ & $8 \mathrm{R}$ \\
\hline \multicolumn{4}{|l|}{ Model $2.6 \% \mathrm{CH}_{4}, 1.4 \% \mathrm{Ar}$} \\
\hline$-\mathrm{N}_{2}+h \vee \rightarrow \mathrm{N}_{2}+(\mathrm{H})$ & & & $7.0 \mathrm{R}$ \\
\hline - photoelectrons $+\mathrm{N}_{2}$ & $0.36 \mathrm{R}$ & $0.6 \mathrm{R}$ & $1.2 \mathrm{R}$ \\
\hline - magnetospheric ${ }^{3} \mathrm{e}^{-}+\mathrm{N}_{2}$ & $\underline{0.06 R^{4}}$ & $\underline{0.08 \mathrm{R}^{4}}$ & $\underline{0.6 R^{4}}$ \\
\hline Total & $0.42 \mathrm{R}$ & $0.68 \mathrm{R}$ & $8.8 \mathrm{R}$ \\
\hline \multicolumn{4}{|l|}{ Model $3.4 \% \mathrm{CH}_{4}, 13.6 \% \mathrm{Ar}$} \\
\hline$\cdot \mathrm{N}_{2}+\mathrm{h} v \rightarrow \mathrm{N}_{2}+(\mathrm{H})$ & & & $6.0 \mathrm{R}$ \\
\hline - photoelectrons $+\mathrm{N}_{2}$ & $3.4 \mathrm{R}$ & $5.7 \mathrm{R}$ & $1.0 \mathrm{R}$ \\
\hline - magnetospheric ${ }^{3} \mathrm{e}^{-}+\mathrm{N}_{2}$ & $\underline{0.5 R^{4}}$ & $\underline{0.84 \mathrm{R}^{4}}$ & $\underline{0.5 \mathbf{R}^{4}}$ \\
\hline Total & $3.9 \mathrm{R}$ & $6.5 \mathrm{R}$ & $7.5 \mathrm{R}$ \\
\hline \multicolumn{4}{|l|}{ Model $5 \% \mathrm{CH}_{4}, 10 \% \mathrm{Ar}$} \\
\hline$\cdot \mathrm{N}_{2}+\mathrm{h} v \rightarrow \mathrm{N}_{2}{ }^{+}(\mathrm{H})$ & & & $6.0 \mathrm{R}$ \\
\hline$\cdot$ photoelectrons $+\mathrm{N}_{2}$ & $3.2 \mathrm{R}$ & $5.2 \mathrm{R}$ & $1.0 \mathrm{R}$ \\
\hline - magnetospheric ${ }^{3} e^{-}+N_{2}$ & $0.5 \mathrm{R}^{4}$ & $\underline{0.82 \mathrm{R}^{4}}$ & $\underline{0.7 \mathrm{R}^{4}}$ \\
\hline Total & $3.7 \mathrm{R}$ & $6.0 \mathrm{R}$ & $7.7 \mathrm{R}$ \\
\hline
\end{tabular}

1 Observations are slit-averaged intensities.

2 Meier et al. [1991] with yield $\phi_{1085}=0.184$.

${ }^{3}$ For power $=2.3 \times 10^{8} \mathrm{~W}$.

${ }^{4}$ Includes $\mathrm{CH}_{4}$ absorption.

and the fact that Strobel and Shemansky were able to fit the Voyager 1 dayside spectrum between 1000-1100 $\AA$ with nitrogen features only. The third model $\left(5 \% \mathrm{CH}_{4}\right.$, $10 \% \mathrm{Ar}$ ) in Table III illustrates that with an elevated vertical mixing rate the Ar mixing ratio at high altitudes can be comparable to a model with a higher tropopause mixing ratio (cf. Fig. 7) and can yield a comparable intensity ratio for Ar lines $/ \mathrm{N}^{-} 1085 \AA$. Thus model atmospheres that are consistent with the UVS solar occultation data but with tropopause $\mathrm{CH}_{4}$ mixing ratios that exceed the IRIS upper limit of 0.034 imply an upper limit on the Ar mixing ratio lower than 0.14. Calculations, not shown in this paper, for bright limb profiles of the Ar resonance lines in comparison with the $\mathrm{N}^{-}(1085-\AA)$ multiplet limb profile yield the same results and conclusions as the disk averaged results in Table III.

\section{CONCLUDING REMARKS}

From this study a firm, basic conclusion is that the slitaveraged absolute intensity of the $\mathrm{N}_{2} c_{4}^{\prime}(0-0,958-\AA)$ plus
$(0-1,981-\AA)$ bands $(\sim 14 R)$ measured by the Voyager 1 UVS during the Titan flyby are inconsistent with model calculations (7-9 $R$ center of disk) based on atmospheres consistent with UVS solar occultation data. The essential problem is that the occultation data require the $\mathrm{CH}_{4}$ mixing ratio to exceed 0.05 above $1000 \mathrm{~km}$, which results in reductions, by factors of 2-4 due to absorption, in the calculated absolute intensity of the $970-\AA$ feature for a pure $\mathrm{N}_{2}$ atmosphere. This inconsistency would be compounded if substantial predissociation occurred in the $\mathrm{N}_{2}$ $c_{4}^{\prime}$ system, but would vanish in the limit of weak predissociation if generous error bars of $\pm 50 \%$ are appropriate for the intensities inferred by Strobel and Shemansky (1982).

Another firm conclusion is that the magnetospheric power dissipation is, at most, $10 \%$ of the solar EUV power input to Titan's extended atmosphere $\left(=2 \times 10^{9} \mathrm{~W}\right.$ for $\lambda<800 \AA$ ). This result is derived from the shape, rather than the absolute intensity, of the bright limb profile for $\mathrm{N}$, emission (924-998 $\AA$ ), and should be independent of calibration and more rigorous if predissociation in the $\mathrm{N}_{2}$ $c_{4}^{\prime}$ system were important. It is consistent with our esti- 
mates of magnetospheric power delivered to the ionopause by curvature drift of thermal and suprathermal electrons and with the downward revision of intensities based on the inflight calibration scheme of Holberg et al. (1982, 1991). Our estimate of magnetospheric power dissipation $\leq 2 \times 10^{8} \mathrm{~W}$ is a factor of 25 less than that inferred by Strobel and Shemansky (1982) and is a factor of 10 less than that inferred by Hunten et al. (1984). The one possible way to solve (1) the shape of the bright limb profile, (2) the absolute intensity problems for disk averaged spectra and bright limb profiles, and (3) the altitude location of the bright limb peak would be the enhancement in the suprathermal, keV electron energy flux relative to the thermal energy flux by more than an order of magnitude. But if this were the explanation, the distinction between the Maxwellian core and the suprathermal tail of the electron energy distribution would vanish.

Likewise our estimate of the nonthermal $\mathrm{N}$ atom escape rate is $\leq 10^{25} \mathrm{sec}^{-1}$ based on detailed $\mathrm{N}_{2}$ dissociation rates by magnetospheric electron and photoelectron impact. Approximately $90 \%$ of the escape is driven by magnetospheric electrons for which we have inferred an upper bound on its magnitude. This estimate is comparable in magnitude to the Monte Carlo calculations of hot $\mathrm{N}$ atom concentrations and fluxes by Lammer and Bauer (1991). There is agreement on the magnitude of the photoelectron contribution, but we favor magnetospheric electrons as the dominant mechanism rather than $\mathrm{N}_{2}^{+}$dissociative recombination.

From the occultation data our preference is for model atmospheres with a tropopause $\mathrm{CH}_{4}$ mixing ratio of $\sim 0.026-0.034$. In order to test the robustness of this conclusion we construct a model atmosphere with a tropopause $\mathrm{CH}_{4}$ mixing ratio of 0.017 and the homopause at $z$ $=875 \mathrm{~km}$. The fit to the optical depth data, not shown, is similar to but slightly worse than the $2.6 \% \mathrm{CH}_{4}$ model atmosphere fit in Fig. 3. The most significant difference between the $1.7 \%$ and $2.6 \%$ models and the $5 \%$ model is the fit to the optical depth profile for the 1151-Å channel. To the extent that this channel indicates the vertical distribution of $\mathrm{CH}_{4}$ density over the altitude range 925-1325 $\mathrm{km}$, the $5 \%$ model is decisively the better fit as it is also for channel $725 \AA$. However, the optical depths range from 0.04 to 4 and the height variations implied by optical depths for channels 928 and $1151 \AA$ are different. The Smith et al. (1982) analysis of the solar occultation data and their derived optical depth profiles are not of sufficient accuracy to extrapolate to the tropopause and infer the $\mathrm{CH}_{4}$ mixing ratio there. Clearly a reanalysis of the Titan UVS solar occultation data is needed to resolve the conflicting optical depth profiles near Ly- $\alpha$ which should be dominated by $\mathrm{CH}_{4}$ absorption.

We conclude that the $\mathrm{CH}_{4}$ mixing ratio from 1100 to $1300 \mathrm{~km}$ is accurately inferred from the solar occultation data (cf. Fig. 7). Although we cannot accurately derive its tropopause value, our preference is for high $\mathrm{CH}_{4}$ mixing ratios and we suggest that the homopause occurs high in the atmosphere, $z \sim 1000 \mathrm{~km}$, with a vertical eddy mixing rate $\sim(4-8) \times 10^{8} \mathrm{~cm}^{2} \mathrm{sec}^{-1}$. An accurate, independent determination of the tropopause $\mathrm{CH}_{4}$ mixing ratio in conjunction with the UVS occultation data could more precisely locate the homopause.

Comparison of the relative intensities of the Ar resonance lines at 1048 and $1067 \AA$ with the $\mathrm{N}^{+}(1085-\AA)$ multiplet yields an upper limit on the Ar mixing ratio of 0.14. Detailed modeling of the UVS airglow spectra similar to the analysis of Strobel and Shemansky (1982) can probably reduce this upper limit to 0.1 .

\section{ACKNOWLEDGMENTS}

We are indebted to D. T. Hall for his assistance and for the computer code used to calculate the UVS slit spatial averaging of model bright limb profiles, to D. J. Strickland for his computer code which was used to calculate magnetospheric electron precipitation, and to A. F. Cheng for helpful discussions. D.F.S. and X.Z. were supported by NASA Grant NAGW-648.

\section{REFERENCES}

Ajello, J. M., G. K. James, B. R. Franklin, and D. E. Shemansky 1989. Medium-resolution studies of extreme ultraviolet emission from $\mathrm{N}_{2}$ by electron impact: Vibrational perturbations and cross sections of the $c_{4}^{\prime \prime} \Sigma_{u}^{+}$and $b^{\prime} 1 \Sigma_{u}^{+}$states. Phys. Rev. A 40, 3524.

ajello, J. M., G. K. James, B. R. Franklin, and D. E. Shemansky 1990. Study of electron impact excitation of argon in the extreme ultraviolet: Emission cross section of resonance lines of Ar I, Ar II. J. Phys. B. 23, 4355-4376.

BATES, D. R. 1959. Some problems concerning the terrestrial atmosphere above about $100 \mathrm{~km}$. Proc. R. Soc. A (London) 253, 451-462.

Broadfoot, A. L., et al. 1981. Extreme ultraviolet observations from Voyager 1 encounter with Saturn. Science 212, 206-210.

CONWAY, R. R. 1983. Multiple fluorescent scattering of $\mathrm{N}_{2}$ ultraviolet emissions in the atmospheres of the Earth and Titan. J. Geophys. Res. 88, 4784-4792.

Flasar, F. M., and B. J. Conrath 1990. Titan's stratospheric temperatures: A case for dynamical inertia? Icarus 85, 346-354.

Fritts, D. C., AND T. J. DUnkerton 1985. Fluxes of heat and constituents due to convectively unstable gravity waves. J. Atmos. Sci.42, $549-556$.

Hall, D. T., D. E. Shemansky, and T. M. Tripp 1992. A reanalysis of Voyager UVS observations of Titan. Paper, Symposium on Titan, Sep. 1991, Toulouse, France, ESA SP-338, 69-74.

Hartle, R. E., E. C. Sittle, JR., K. W. Ogilvie, J. D. Scudder, A. J. Lazarus, and S. K. Atreya 1982. Titan's ion exosphere observed from Voyager 1. J. Geophys. Res. 87, 1383-1394.

Hill, T. W., A. J. Dessler, and C. K. Goertz 1983. Magnetospheric models. In Physics of the Jovian Magnetosphere (A. J. Dessler, Ed.), pp. 353-394. Cambridge Univ. Press, New York.

HINson, D. P., AND G. L. TYLER 1983. Internal gravity waves in Titan's atmosphere observed by Voyager radio occultation. Icarus $\mathbf{5 4}$, 337-352.

Hinteregger, H. E., K. Fukui, And B. R. Gilson 1981. Observa- 
tional, reference and model data on solar EUV, from measurements on AE-E. Geophys. Res. Lett. 8, 1147-1150.

Holberg, J., W. T. Forrester, and D. E. Shemansky 1982. Voyager absolute far-ultraviolet spectrophotometry of hot stars. Astrophys. $J$. 257, 656-671.

Holberg, J. B., B. Ali, T. W. Carone, and R. S. Polidan 1991. Absolute far-ultraviolet spectrometry of hot subluminous stars from Voyager. Astrophys. J. 375, 716-721.

Hubbard, W. B., D. M. Hunten, H.J. Brosch, Y. Nevo, E. CarREIRA, F. Rossi, AND L. H. WASSERMAN 1990. Results for Titan's atmosphere from its occultation of 28 Sagittarii. Nature 343, 353-355.

Hunten, D. M., et al. 1984. Titan. In Saturn (T. Gehrels and M. S. Matthews, Eds.), pp. 671-759. Univ, of Arizona Press, Tucson.

IP, W.-H. 1990. Titan's upper ionosphere. Astrophys. J. 362, 354-363.

James, G. K., J. M. Ajello, B. Franklin, and D. E. Shemansky 1990. Medium resolution studies of extreme uv emission from $N_{2}$ by electron impact: The effect of predissociation on the emission cross section of the $b^{i} \Pi_{u}$ state, J. Phys. B. 23, 2055.

LAMmer, H., AND S. J. BAUER 1991. Nonthermal atmospheric escape from Mars and Titan. J. Geophys. Res. 96, 1819-1825.

Lellouch, E., A. Coustenis, D. Gautier, F. Raulin, N. Dubouloz, AND C. Frere 1989. Titan's atmosphere and hypothesized ocean: A reanalysis of the Voyager 1 radio-occultation and IRIS 7.7- $\mu \mathrm{m}$ data. Icarus 79, 328-349.

LinDzEN, R. S. 1981. Turbulence and stress owing to gravity wave and tidal breakdown. J. Geophys. Res. 86, 9707-9714.

Maclennan, C. G., L. J. Lanzerotti, S. M. Krimigis, R. P. Lepping, AND N. F. Ness 1982. Effects of Titan on trapped particles in Saturn's magnetosphere. J. Geophys. Res. 87, 1411-1418.

Meier, R. R., J. A. R. Samson, Y. Chung, E.-M. Lee, and Z.-X. He 1991. Production of $\mathrm{N}^{+*}$ from $\mathrm{N}_{2}+h v$ : EUV emission yields from laboratory and dayglow data. Planet. Space Sci. 39, 1197-1208.

Neubauer, F. M., D. A. Gurnett, J. D. Scudder, and R. E. Hartile 1984. Titan's magnetospheric interaction. In Saturn (T. Gehrels and M. S. Matthews, Eds.), pp. 760-787. Univ. of Arizona Press, Tucson.
Samuelson, R. E., R. A. Hanel, V. G. Kunde, and W. C. Maguire 1981. Mean molecular weight and hydrogen abundance of Titan's atmosphere. Nature 292, 688-693.

SiCARDY, B., et al. 1990. Probing Titan's atmosphere by stellar occultation. Nature 343, 350-352.

Smith, G. R., D. F. Strobel, A. L. Broadfoot, B. R. Sandel, D. E. SHEMANSKY, AND J. B. Holberg 1982. Titan's upper atmosphere: Composition and temperature from the EUV solar occultation. $J$. Geophys. Res. 87, 1351-1359.

Steiner, G., AND S. J. BAUER 1990. Molecular and eddy diffusion in the atmosphere of Titan. Ann. Geophys. (Gauthier-Villars) 8, 473-476.

Strickland, D. J., AND R. R. MEIER 1982. A photoelectron model for the rapid computation of atmospheric excitation rates. NRL Memorandum Report 5004

Strickland, D. J., R. R. Meier, J. H. Hecht, and A. B. Christensen 1989. Deducing composition and incident electron spectra from ground-based auroral optical measurements: Theory and model results. J. Geophys. Res. 94, 13.527.

STrobel, D. F. 1989. Constraints on gravity wave induced diffusion in the middle atmosphere. Pure Appl. Geophys. 130, 533-546.

Strobel. D. F., and D. E. Shemansky 1982. EUV emission from Titan's upper atmosphere: Voyager 1 encounter. J. Geophys. Res. 87, 1361-1368.

Strobel, D. F., J. P. Apruzese, and M. R. Schoeberl 1985. Energy balance constraints on gravity wave induced eddy diffusion in the mesosphere and lower thermosphere. J. Geophys. Res. 90, $13,067-13,072$.

Strobel, D. F., A. F. Cheng, M. E. Summers, and D. J. Strickland 1990. Magnetospheric interaction with Triton's ionosphere. Geophys. Res. Lett. 17, 1661-1664.

Strobel, D. F., R. R. Meier, M. E. Summers, and D. J. Strickland 1991. Nitrogen airglow sources: Comparison of Triton, Titan, and Earth. Geophys, Res, Lett. 18, 689-692.

YELLE. R. V. 1991. Non-LTE models of Titan's upper atmosphere. Astrophys. J, 383, 380-400.

Yung, Y. L., M. Allen, And J. P. Pinto 1984. Photochemistry of the atmosphere of Titan: Comparison between model and observations. Astrophys. J. Supp. 55, 465-506. 